\title{
Nociceptin/orphanin FQ opioid receptor (NOP) selective ligand MCOPPB links anxiolytic and senolytic effects
}

\author{
Marco Raffaele • Kristina Kovacovicova • Tommaso Biagini • Oriana Lo Re • Jan Frohlich • \\ Sebastiano Giallongo · James D. Nhan • Antonino Giulio Giannone $\cdot$ Daniela Cabibi $\cdot$ Martin Ivanov \\ Anton B. Tonchev $\cdot$ Martin Mistrik • Matthew Lacey $\cdot$ Petr Dzubak $\cdot$ Sona Gurska $\cdot$ Marian Hajduch • \\ Jiri Bartek • Tommaso Mazza • Vincenzo Micale · Sean P. Curran • Manlio Vinciguerra (D)
}

Received: 11 October 2021 / Accepted: 5 November 2021 / Published online: 24 November 2021

(C) The Author(s), under exclusive licence to American Aging Association 2021

\begin{abstract}
Accumulation of senescent cells may drive age-associated alterations and pathologies. Senolytics are promising therapeutics that can preferentially eliminate senescent cells. Here, we performed a high-throughput automatized screening (HTS) of the commercial LOPAC®Pfizer library on aphidicolin-induced senescent human fibroblasts, to
\end{abstract}

Marco Raffaele and Kristina Kovacovicova contributed equally to this work

Supplementary Information The online version contains supplementary material available at https://doi. org/10.1007/s11357-021-00487-y.

M. Raffaele $\cdot$ K. Kovacovicova $\cdot$ O. Lo Re $\cdot$ J. Frohlich $\cdot$

S. Giallongo $\cdot$ M. Vinciguerra

International Clinical Research Center, St. Anne's

University Hospital, Brno, Czech Republic

K. Kovacovicova

Psychogenics Inc, Tarrytown, NY, USA

T. Biagini · T. Mazza

Laboratory of Bioinformatics, Fondazione IRCCS Casa

Sollievo Della Sofferenza, San Giovanni Rotondo, Italy

O. Lo Re $\cdot$ A. B. Tonchev $\cdot$ M. Vinciguerra $(\bowtie)$

Department of Translational Stem Cell Biology, Research

Institute of the Medical University of Varna, Varna,

Bulgaria

e-mail: manlio.vinciguerra@fnusa.cz

S. Giallongo

Department of Biology, Faculty of Medicine, Masaryk

University, Brno, Czech Republic identify novel senolytics. We discovered the nociceptin receptor FQ opioid receptor (NOP) selective ligand 1-[1-(1-methylcyclooctyl)-4-piperidinyl]-2[(3R)-3-piperidinyl]-1H-benzimidazole (MCOPPB, a compound previously studied as potential anxiolytic) as the best scoring hit. The ability of MCOPPB to eliminate senescent cells in in vitro models was further tested in mice and in C. elegans. MCOPPB reduced the senescence cell burden in peripheral tissues but not in the central nervous system. Mice and worms exposed to MCOPPB also exhibited locomotion and lipid storage changes. Mechanistically, MCOPPB treatment activated transcriptional

\section{J. D. Nhan · S. P. Curran \\ Leonard Davis School of Gerontology, University of Southern California, Los Angeles, CA, USA}

\section{J. D. Nhan · S. P. Curran}

Department of Molecular and Computational Biology, Arts, and Sciences, Dornsife College of Letters, University of Southern California, Los Angeles, CA, USA
A. G. Giannone - D. Cabibi
Department of Health Promotion, Mother and Child Care, Internal Medicine and Medical Specialties, Pathologic Anatomy Unit-University of Palermo, Palermo, Italy
M. Ivanov · A. B. Tonchev
Department of Anatomy and Cell Biology, Research Institute of the Medical University of Varna, Varna, Bulgaria 
networks involved in the immune responses to external stressors, implicating Toll-like receptors (TLRs). Our study uncovers MCOPPB as a NOP ligand that, apart from anxiolytic effects, also shows tissue-specific senolytic effects.

\section{Keywords Senolytic $\cdot$ NOP $\cdot$ Senescence $\cdot$ Aging}

\section{Introduction}

The majority of chronic diseases appears with increasing age and thus has a high prevalence in the elderly [1, 2]. Similarly, geriatric conditions, such as mild cognitive impairment and frailty, drastically augment with the aging of the individual [3]. A great bulk of evidence shows that the progressive accumulation of senescent cells can mark and drive ageassociated alterations and pathologies [4-6]. Cellular senescence is characterized by a stable growth arrest and other phenotypic alterations, including upregulation of tumor suppressor p16 and a pro-inflammatory secretome (senescence-associated secretory phenotype, SASP) [7]. At the same time, senescence plays roles in normal development, maintains tissue homeostasis, and limits the onset of tumorigenesis [8]. Rapid gain of interest in cellular senescence is rising from the potential of therapeutic targeting of this process to improve obesity, age-related pathologies, and

M. Mistrik · M. Lacey · P. Dzubak · S. Gurska - M. Hajduch · J. Bartek

Institute of Molecular and Translational Medicine, Faculty of Medicine and Dentistry, Palacky University, Olomouc, Czech Republic

\section{J. Bartek}

Genome Integrity Unit, Danish Cancer Society Research Center, Copenhagen, Denmark

\section{J. Bartek}

Division of Genome Biology, Department of Medical Biochemistry and Biophysics, Science for Life Laboratory, Karolinska Institute, Stockholm, Sweden

V. Micale

Department of Biomedical and Biotechnological Sciences, University of Catania, Catania, Italy

\section{S. P. Curran}

Norris Comprehensive Cancer Center, Keck School of Medicine, University of Southern California, Los Angeles, CA, USA as adjuvant tumor therapy, using drugs called senolytics [9-13]. Known senolytic agents include the first and best-studied cocktail dasatinib (D) plus quercetin (Q), discovered using a mechanism-based approach [14], and others [9-11].

A great help in the research of new effective compounds can be given by using high-throughput automatized screening (HTS) technology. This approach allows testing thousands of molecules present in the market as chemical libraries [15], in in vitro senescence models, increasing the chance to identify new potential senolytics. Recently, HTS on commercial chemical libraries led to discover two new families of senolytics, HSP90 inhibitors [16] and BET family protein degraders (BETd) [17], respectively. In these studies, as well as others, fibroblast cells were mainly used, such as human dermal fibroblasts (HDFs) [18], IMR90 [17], MRC5, and BJ [19], or the murine embryonic fibroblasts (MEFs) [16], under treatment with antiproliferative and DNA damaging agents to induce senescence. The tetracyclic diterpenoid antibiotic aphidicolin (APH), a specific inhibitor of B-family DNA polymerases [20, 21], is a potent inducer of senescence in various experimental in vitro setups (reviewed in (32)). Low doses of APH are generating substantial replication stress accompanied by chromosomal breaks within common fragile sites [22] and widespread changes in both gene expression and protein phosphorylation patterns leading to the development of senescence in many cell lines [23-26]. In the present study, we screened the commercially available library of pharmacologically active compounds LOPAC®Pfizer - at a fixed $10 \mu \mathrm{M}$ concentration - on aphidicolin-induced senescence in human MRC5 fibroblast cells, in an attempt to identify new senolytics. From the library screening, we identified the nociceptin receptor agonist MCOPPB as a potent senolytic compound. Further experiments confirmed the ability of MCOPPB to eliminate senescent cells in vitro and reduce the senescence cell burden in vivo.

\section{Materials and methods}

Cell culture

HepG2 and Huh-7 cell lines were obtained from CLS-GmbH (Eppelheim, Germany) and MRC5 cell line from ATCC. All cell lines were cultured in 
DMEM (1X) supplemented with $10 \%$ fetal bovine serum (FBS), with $1 \%$ penicillin/streptomycin. For chemotherapy-inducing senescence experiments, cells were treated for $24 \mathrm{~h}$ with $100 \mathrm{nM}$ Doxorubicin (Sigma-Aldrich, MO, USA) dissolved in DMSO. After $24 \mathrm{~h}$, the cells were treated with MCOPPB at different concentrations for $48 \mathrm{~h}$ and then fixed in paraformaldehyde $2 \%+$ glutaraldehyde $0.5 \%$, washed in PBS twice, and used for the X-gal assay. After counterstain with Nuclear Fast Red, the coverslips were dehydrated with an increasing concentration of ethanol $(50,70,95,100 \%)$, cleared in xylene for $20 \mathrm{~min}$, and mounted in DPX. Cell viability was assessed by using alamarBlue ${ }^{\mathrm{TM}}$ Cell Viability Reagent (Thermo Scientific, MA, USA), according to the manufacturer's instructions.

\section{HTS screening}

To obtain a large quantity of MRC5 fibroblasts, the cells were cultivated in the presence of $0.2 \mu \mathrm{M}$ of aphidicolin (Sigma-Aldrich, MO, USA). Cells were regularly divided half to half once reaching the full confluence for approximately 4 weeks when the population growth stopped completely. Normal proliferating and senescent MRC5 cells were seeded into 384-well plates (SpectraPlate, PerkinElmer, MA, USA) in density 2000 cells per well. For the screening, we used a library of small chemical compounds that contained 90 commercial drugs and was developed by Pfizer (LOPAC®Pfizer, Cat. No: LO5100; Sigma-Aldrich, MO, USA). Tested compounds were dissolved in $100 \%$ DMSO and $24 \mathrm{~h}$ after seeding the cells were treated by selected compounds in fixed $10 \mu \mathrm{M}$ concentration by echo acoustic non-contact liquid handler Echo 550 (Labcyte, CA, USA), each compound in triplicate. The toxic response was evaluated 5 days after the treatment using XTT assay (AppliChem, Darmstadt, Germany) according to the instructions provided by the manufacturer. Briefly, medium containing the library drugs was removed, and the cells were incubated with medium containing XTT salts for $3 \mathrm{~h}$. Absorbance of the wells was measured at $492 \mathrm{~nm}$ using the FLUOstar Optima FL plate reader (BMG Labtech, Ortenberg, Germany), and the results were expressed as the average of percentage of inhibition (AVG PI) compared to the non-treated cells.
SA-beta-gal staining of cells

For cellular senescence confirmation was used $\beta$-galactosidase staining kit (Cell Signalling Technology \#9860S; MA, USA) according to the manufacturer's protocol. Briefly, cells were washed with $1 \times \mathrm{PBS}$ and fixed at room temperature for $10 \mathrm{~min}$ by enclosed fixative and washed with $1 \times$ PBS twice. Following fixation, the enclosed $\beta$-galactosidase staining solution was added, and the samples were incubated at $37{ }^{\circ} \mathrm{C}$ overnight. After staining, the stained cells were examined and/or photographed using a transmission light microscope (Primo Vert, Zeiss, Oberkochen, Germany) using $20 \times$ or $40 \times$ objectives.

Immunofluorescence staining of cell lines

The cells were fixed with $10 \%$ formalin for $10 \mathrm{~min}$ directly on the cultivation dishes (TPP) and washed by PBS. Small disks (approx. $1.2 \mathrm{~cm}$ in diameter) were cut from the dishes using an in-house build cutter. Samples were stained with primary antibodies at $4{ }^{\circ} \mathrm{C}$ overnight, then with secondary antibodies at room temperature for $1 \mathrm{~h}$, and finally incubated in $1 \mu \mathrm{g} / \mathrm{ml}$ DAPI solution in water at room temperature for $5 \mathrm{~min}$ before mounting. After mounting, the images were acquired using a fluorescence microscope (Zeiss LSM780 with Elyra PS.1 module) using a $63 \times$ objective. The following primary antibodies were used: $\gamma \mathrm{H} 2 \mathrm{AX}$ (pSer139, 500×, 07-146, Millipore, MA, USA), 53BP1 (SC22760, 300×, Santa Cruz, CA, USA). Secondary anti-mouse and anti-rabbit antibodies were Alexa Fluor 488 and Alexa Fluor $568(1000 \times$, Invitrogen, MA, USA).

\section{CF12FDG staining}

Cellular senescence in HepG2 and Huh-7 cells was quantified using CF12FDG [5-dodecanoylaminofluorescein Di- $\beta$-D-galactopyranoside] (Satereh Biotech, OR, USA). CF12FDG is a non-fluorescent, lipophilic, beta-galactosidase substrate. The substrate is cleaved by $\beta$-galactosidase producing a fluorescent product that is well retained by the cells. CF12FDG detection was performed by flow cytometry as previously described [27]. Briefly, lysosomal alkalinization of HepG2 or Huh-7 cells was induced by incubation of Baf A1 (100 nM) in humidified air with $5 \% \mathrm{CO} 2$ 
at $37 \mathrm{C}$ for $1 \mathrm{~h}$. CF12FDG was added to the culture media containing Baf A1 for another $2 \mathrm{~h}$. After fixation with $4 \%$ formaldehyde at room temperature, nuclei were stained with DAPI. To quantify the cells positive for CF12FDG, cells were rinsed with PBS, trypsinized, collected, resuspended in ice-cold PBS, and analyzed using a flow cytometer FACS Canto (BD Biosciences, NJ, USA).

\section{Mice models}

All experiments were carried out following the rules of reduction of numbers of animals and minimizing their suffering during the experiments. MCOPPB for experimentation was purchased from Sigma-Aldrich (PZ0159, MO, USA). All animal work was conducted either according to Act No 246/1992 Coll., on the protection of animals against cruelty, or according to animal license number BMBWF66.015/0037/ V/3b/2019 and to Austrian legislation. The MCOPPB administration started at the age of 20 weeks according to the following scheme: the i.p injection for 5 consecutive days and 2 day rest period over 3 weeks. The initial dose was $5 \mathrm{mg} / \mathrm{kg}$ for the first week and $2.5 \mathrm{mg} / \mathrm{kg}$ for the subsequent 2 weeks. Upon sacrifice at the 28th week, blood samples were collected and used for blood cells count, while organs were harvested and processed for the histological analysis.

\section{Behavioral studies}

C57/BL6 male mice (Charles River) were transferred to the preclinical phenotyping facility of the Vienna Biocenter Core Facilities GmbH (VBCF) about 2 months before injections and housed at a 14-h light/10-h dark cycle in IVC racks with access to food and water ad libitum. Behavioral experiments were conducted in battery with 1 day in between two consecutive tests, in the following order: (a) exploratorybased approach-avoidance conflict tests, open-field test, elevated plus maze, spontaneous alternation in the Y-maze; (b) depressive-like behavior paradigms, forced swim test; (c) PhenoMaster measurements. The order of tests within the battery was designed based on previous testing experience [28]. Before each experiment, mice were allowed to habituate to the experimental room for at least 30 min before any testing. Experiments $\mathrm{a}-\mathrm{b}$ were performed between 8 am and $1 \mathrm{pm}$ (lights on) during the day.
Open field: Naïve mice were allowed to explore an open field arena (www.tse-systems.com) sized $50 \mathrm{~cm}$ (width) $\times 50 \mathrm{c} \mathrm{m}$ (length) $\times 29.5 \mathrm{~cm}$ (height) for $30 \mathrm{~min}$ with the release from the center and video tracked using TSE VideoMot 3D version 7.01 software as previously described [28, 29]. In the software, a "center" zone was defined as a central square $25 \mathrm{~cm} \times 25 \mathrm{~cm}$ in size, the rest being the "border zone." Light conditions were about $100 \mathrm{~lx}$ in the center zone. The time spent in each zone, distance traveled, number of center visits, and rearings were recorded as readout parameters.

Elevated plus-maze: Mice were placed in the center zone $(6.5 \times 6.5 \mathrm{~cm})$, facing an open arm of a custom-built elevated plus maze (elevated $54 \mathrm{~cm}$ above the floor) with 2 open arms (OA, $30 \mathrm{~cm}$ length, $7 \mathrm{~cm}$ width) and 2 wall-enclosed arms (closed arms, CA, $30 \mathrm{~cm}$ length, $6 \mathrm{~cm}$ width, walls $14.5 \mathrm{~cm}$ high) and let explore freely for $5 \mathrm{~min}$, as previously described [28, 30]. Their path was video tracked using TopScan software (CleverSys, Inc., VA, USA), and the amount of time spent and distance traveled in the open arms, closed arms, and the center zone was evaluated. Lux levels were about $180 \mathrm{~lx}$ in the center zone and open arms and about $35 \mathrm{~lx}$ in closed arms.

Y-maze: The Y-maze was performed as a test for working memory using a custom-built Y-shaped maze (made by the workshop of Research Institute of Molecular Pathology (IMP) and Institute of Molecular Biotechnology (IMBA), Austrian Academy of Sciences, Vienna, Austria) with grey, opaque walls, and floor and the following dimensions: arm length, $30 \mathrm{~cm}$; arm width, $6 \mathrm{~cm}$; wall height, $14.5 \mathrm{~cm}$. After $30 \mathrm{~min}$ of habituation to the test room (lux levels approx. $180 \mathrm{~lx}$, visual cues on walls), mice were placed individually into the end of one of the 3 arms ( $\operatorname{arm~A,~B,~C),~facing~the~wall~at~the~end~of~}$ the arm, and let explore the maze for $5 \mathrm{~min}$ while being video tracked with TopScan software (CleverSys, Inc., USA) [31]. The experimenter watched the videos in the same room, behind a curtain, and scored the latency to leave the starting arm (which was alternated between the mice) and the number and sequence of arm entries. This sequence was evaluated in terms of triplets. Three arm entries in a row were scored as correct spontaneous alternations (SA; e.g., $\mathrm{BAC}, \mathrm{CBC}, \mathrm{ABC}$ ), erroneous alternate arm returns (AARs; e.g., BAB, $\mathrm{CBC}, \mathrm{ABA}$ ), or erroneous same arm returns (SARs, e.g., BAA, CCB, AAC). After 
each triplet was scored, the start of the analysis was shifted by 1 entry, and the next triplet sequence was scored. Two such shifts of the analysis result in overlapping triplets and the scoring of all possible decision points of the mouse. Spontaneous alternation performance (SAP) was calculated as [spontaneous alternations (SA) / (total arm entries - 2)].

Forced swim test: The forced swim test (FST) employed here was essentially similar to that described elsewhere [32-34]. Up to 4 mice were placed in 2-1 glass beakers $(14 \mathrm{~cm}$ diameter, $20 \mathrm{~cm}$ height), visually separated from each other, and filled with tap water at room temperature $\left(24{ }^{\circ} \mathrm{C}\right)$ up to a level of $11 \mathrm{~cm}$. They were filmed for 6 min while struggling/swimming/floating using a Sony video camera and then let dry under an infrared lamp for a few minutes in their home cage. After the vigorous activity, swimming attempts cease, and the animal adopts a characteristic immobile posture. A mouse is judged to be immobile when it floats in an upright position and makes only small movements to keep its head above water. The duration of mobility was recorded during the last 4-min of the 6-min testing period. All trials were recorded for subsequent offline analysis.

PhenoMaster measurement: mice were habituated to PhenoMaster drinking nozzles using training bottles provided by TSE Systems group-housed in their home cage for 3 days and then single-housed (with training bottles) before being placed in the TSE PhenoMaster with a climate chamber (https:// www.tse-systems.com/product-details/phenomaster) for 8 days under the same 14:10 light cycle as in the animal facility at $24{ }^{\circ} \mathrm{C}$ and $50 \%$ humidity of the air. The set-up of the PhenoMaster allowed for the circadian measurement of the amount of food and water consumed, the locomotion activity in the cage (using AciMot activity frames), the amount of oxygen consumed, and $\mathrm{CO}_{2}$ produced (with gas sampling values every $15 \mathrm{~min}$, set at an airflow of $0.5 \mathrm{l} / \mathrm{min}$ ) and thus allowed the calculation of the respiratory exchange rate RER and the consumed calories.

Histology and immunohistochemistry

Samples of liver and fat tissues were harvested from euthanized mice, fixed with $4 \%$ paraformaldehyde, processed for the embedding in OCT or paraffin wax, and sectioned for histopathological analysis.
The paraffin liver sections were stained with hematoxylin and eosin (H\&E) for the overall assessment of parenchymal architecture, hepatocyte abnormalities (including ballooning), inflammatory infiltration, and the measurement of lipid droplet size. These features were scored according to the NAFLD histologic activity score (NAS) system [35] Briefly, double-blinded analysis identified the score of steatosis (grade $0 \leq 5 \% ; 1=5-33 \%$; grade $2=34 \%-66 \%$; grade $3 \geq 66 \%$ ), lobular inflammation ( $0:$ no foci, $1:<2$ foci per $200 \times$ field, $2: 2$ to 4 foci per $200 \times$ field, and $3:>4$ foci per $200 \times$ field), hepatocyte ballooning ( 0 : none; 1: rare or few; 2: many). Periodic acid-Schiff (PAS) staining was performed for the evaluation of glycogen content. All analyses were performed in triplicate by two independent pathologists.

Immunofluorescence staining of mice tissues

Immunofluorescence staining was performed on mice tissues sections as previously described [36]. Briefly, mice tissue sections were re-hydrate in PBS for $10 \mathrm{~min}$ and treated with the TrueBlack Autofluorescence quencher (Biotium, CA, USA) for $30 \mathrm{~s}$. After careful washing in PBS, sections were blocked for $60 \mathrm{~min}$ in M.O.M blocking solution (Vector Laboratories, CA, USA) and then incubated with primary antibody overnight. Two primary antibodies $\beta$-gal (ab9361, Abcam, Cambridge, UK) and F4-80 (ab6640, Abcam, Cambridge, UK) were mixed for co-staining liver sections. All the antibodies were used at 1:500 dilution. The staining was developed using Alexa fluorescent $(488,647)$ conjugated secondary antibodies, and images were acquired using Axio scan Z1 (Zeiss, Oberkochen, Germany).

$\mathrm{X}$-gal staining of tissues

The $\beta$-galactosidase detection method was performed as previously described [36]. Briefly, tissues frozen sections were fixed in $1 \%$ formalin in PBS for $1 \mathrm{~min}$ at RT, washed three times in PBS, and incubated overnight on X-gal staining solution $[1 \mathrm{mg} / \mathrm{mL}$ of X-gal (VWR, PA, USA), $40 \mathrm{mM}$ citric acid/sodium phosphate buffer, $5 \mathrm{mM}$ potassium ferricyanide (Sigma-Aldrich, MO, USA), $5 \mathrm{mM}$ potassium ferrocyanide (Sigma-Aldrich, MO, USA), $150 \mathrm{mM} \mathrm{NaCl}$, and $2 \mathrm{mM} \mathrm{MgCl} 2$ ] at $37{ }^{\circ} \mathrm{C}$ in a humidified chamber. The experiments were carried out using staining 
solutions at $\mathrm{pH} 6.0$ to assess the SA- $\beta$-gal activity. Samples were rinsed with distilled water and counterstained with Nuclear Fast Red (Sigma-Aldrich, MO, USA) for 5 min. Images were acquired using PiaApochromat $20 \times 0.8 \mathrm{M} 27$ objective on Axio scan Z1 (Zeiss, Oberkochen, Germany).

\section{RNA-Seq}

Total RNA was extracted from the liver of control and treated mice using TRIzol Reagent (Thermo Fisher Scientific, MA, USA). Indexed libraries were prepared from $250 \mathrm{ng}$ purified RNA using the NEBNext Ultra II Directional RNA Library preparation kit with polyA selection module (New England Biolabs, MA, USA). Libraries and input RNA samples were quality checked using Fragment Analyzer (Advanced Analytical, IA, USA). The libraries were pooled so that each index-tagged sample was present in equimolar amounts. The pooled samples then underwent cluster generation and sequencing using an Illumina NextSeq 500 (Illumina, CA, USA) in a $2 \times 75$ paired-end format. Short reads were aligned against the GRCm38.p6 genome assembly, using STAR (ver. 2.6.1a). Piled-up reads were counted with htseq-count [37]. Read-count normalization and comparisons were performed using the edgeR R package. Genes were considered differentially expressed between groups if their expression values significantly differed by $\geq 2$ folds. Correction for multiple tests was achieved by the Benjamini-Hochberg procedure. The significance threshold was set to 0.05 . Functional and pathway enrichment analyses were performed using the Ingenuity Pathway Analysis (QIAGEN Inc.). An interaction network was built using STRING (https://string-db.org). The RNASeq profiling data have been deposited in GEO with the accession number GSE161412. This dataset was compared with three publically available genomewide expression profiles of young and old mouse liver retrieved from the Gene Expression Omnibus database (GSE57809, GSE113745, and GSE104395). Read counts were retrieved as TPM values (Transcripts Per Kilobase Million) and compared gene-wise between young and old mice using moderated $t$ tests (mod.t.test function of the MKmisc R Bioconductor package). Genes were considered differentially expressed (DEG) between contrasts if Benjamini-Hochberg-adjusted $p$ values $<0.05$ and $\mid \log 2$ (fold-change) $\mid>1$. The datasets were then pooled with samples of this study, and a unique matrix of normalized read counts for 15,662 shared genes among all was created. Batch effects were reduced using the ComBat function of the SVA $\mathrm{R}$ package, and then samples were quintile-normalized (normalize.quantiles function of the preprocessCore $\mathrm{R}$ package). We then sought for the optimal number of clusters of samples within the preprocessed matrix of counts using the implementation of the silhouette method of fviz_nbclust function of the factoextra $\mathrm{R}$ package. Samples were then subjected to $k$-means partitioning, where the value of $k$ was deduced from the previous step. The distribution of subjects into clusters was represented by ggplot2 $\mathrm{R}$ package.

C. elegans maintenance and growth

Wild-type N2 Bristol strain was used for all experiments. Worms were raised on NGM agar plates, seeded with $250 \mu \mathrm{L}$ of $E$. coli (strain OP50), at $20^{\circ} \mathrm{C}$. Age synchronous larval stage 1 (L1) was used for the beginning of all experiments and was attained by hypochlorite treatment of adults to collect eggs and allowing them to hatch overnight while rocking in minimal media (M9).

MCOPPB treatment of C. elegans

10X OP50: OP50 bacteria were grown overnight in LB with streptomycin. The following day, the bacteria were spun down at $4500 \mathrm{rcf}$ for $20 \mathrm{~min}$ at $4{ }^{\circ} \mathrm{C}$, and the supernatant was removed without disturbing the pellet. Enough M9 to make 10 times concentrated OP50 was used to resuspend the pellet, which can be stored at $4{ }^{\circ} \mathrm{C}$ for later use. MCOPPB: $1 \mathrm{mg}$ of MCOPPB trihydrochloride (Sigma-Aldrich, MO, USA) was dissolved in $1 \mathrm{~mL}$ of autoclaved water for a final concentration of $1.8725 \mathrm{mM}$ and was freshly made before every experiment. For liquid NGM, $3 \mathrm{~g} \mathrm{NaCl}$ and $2.5 \mathrm{~g}$ of peptone are mixed with $970 \mathrm{~mL}$ of nanopure water and autoclaved for $1 \mathrm{~h}$. The mixture is then allowed to mix and cool down to $55^{\circ} \mathrm{C}$. Following cooling, $1 \mathrm{~mL}$ of cholesterol $\left(5 \mathrm{mg} / \mathrm{mL}\right.$ in ethanol), $1 \mathrm{~mL}$ of $1 \mathrm{M} \mathrm{CaCl}_{2}$, $1 \mathrm{~mL}$ of $1 \mathrm{M} \mathrm{MgSO}_{4}$, and $25 \mathrm{~mL}$ of $1 \mathrm{M} \mathrm{KO}_{4}(\mathrm{pH}$ 6.0) are added to the mixture. All experiments were set up in the same manner and set up to be used in liquid culture. For each condition in each experiment, a master mix containing $100 \mathrm{~L} 1$ synchronized worms/ $\mathrm{mL}, 60 \mu \mathrm{L} / \mathrm{mL}$ of $10 \mathrm{X}$ concentrated OP50, $17.6 \mu \mathrm{L} /$ $\mathrm{mL}$ of either $1.8725 \mathrm{mM}$ MCOPPB for treatment or 
autoclaved water for control, and the rest liquid NGM to bring to a final volume of master mix.

\section{C. elegans Nile red staining}

The experiment was done in a 24 well cell culture plate, with $600 \mu \mathrm{L}$ of worm master mix in each well for each condition. Each well represents a separate replicate. A cover film was placed over the plate before putting on the lid to prevent evaporation. The plate was rocked in a $20{ }^{\circ} \mathrm{C}$ incubator for $48 \mathrm{~h}$ to allow worms to reach larval stage 4 (L4) to stain for lipids. Nile red staining and analysis were performed as previously described [38].

\section{C. elegans lifespan}

Experimental procedure: The experiment was done in a 96 well cell culture plate, $1 \mathrm{~mL}$ of worm master mix was made for each row in triplicate for either control or treatment, and $120 \mu \mathrm{L}$ of each master mix was dispensed into each well for eight wells total for each row. $7.5 \mu \mathrm{L}$ of $2.5 \%$ streptomycin was added to each $1 \mathrm{~mL}$ master mix to prevent contamination for the extended experiment. The plate was covered with plastic film and was rocked in a $20{ }^{\circ} \mathrm{C}$ incubator for the duration of the experiment. Forty-eight hours after the start of the experiment, $30 \mu \mathrm{L}$ of $0.6 \mathrm{mM}$ FUDR was added to each well to inhibit the growth of progeny. Seven days after the start of the experiment, $5 \mu \mathrm{L}$ of the 10X OP50 was added to each well to supplement the food and prevent starvation.

Lifespan scoring: The survival of worms was checked every 2 days for the duration of the experiment. During each counting day, the plastic film cover is removed, and the plate is vigorously shaken to count and aerate the worms. Any wells with more than 18 worms were censored as an excess of worms can cause dietarily restricted-like phenotypes. Worms were only marked dead if after vigorous shaking they did not move. Some worms did not properly develop into adulthood following FUDR treatment and were censored as well.

\section{C. elegans qPCR}

Experiment set-up: The experiment was done in a 24 well plate, with $900 \mu \mathrm{L}$ of worm master mix per well, and each $1 \mathrm{~mL}$ of master mix supplemented with $7.5 \mu \mathrm{L}$ of $2.5 \%$ streptomycin. All 24 wells were full of worms, with one plate being for control and another for treatment. Plates were rocked in a $20{ }^{\circ} \mathrm{C}$ incubator for $48 \mathrm{~h}$ and subsequently collected for RNA. Each row of wells for each plate was pooled together for one replicate, so six wells per replicate. Following collection, worms were allowed to gravity settle, the supernatant was collected without disturbing the pellet, and fresh M9 was added. This step was repeated 3 times. Following the last wash step, all the media was removed without disturbing the pellet, and $500 \mu \mathrm{L}$ of TRIzol was added, and worms were stored at $-80^{\circ} \mathrm{C}$ until RNA was ready to collect.

RNA collection: Frozen worms were thawed in TRIzol and spun down for $1 \mathrm{~min}$ at 14,000 rcf to pellet worms. TRIzol was removed, and $500 \mu \mathrm{L}$ of water was added to wash worms. Pellet was vortexed to dislodge, and the washing step was repeated two more times. Following the last removal of the wash, $300 \mu \mathrm{L}$ of worm lysis buffer (50 mM KCl, $10 \mathrm{mM}$ Tris ( $\mathrm{pH} 8.0$ ), $2.5 \mathrm{mM} \mathrm{MgCl}$, 0,45\% IGEPAL CA-630, 0,45\% Tween-20, 0.01\%(w/v) gelatin, $60 \mu \mathrm{g} / \mathrm{mL}$ proteinase $\mathrm{K}$ ) was added to resuspend pellet. Worms were then lysed in a $65{ }^{\circ} \mathrm{C}$ heat block for $10 \mathrm{~min}$, followed by inactivation of proteinase $\mathrm{K}$ at $95{ }^{\circ} \mathrm{C}$ for $1 \mathrm{~min}$. The lysis solution was then spun down at 14,000 rcf for $20 \mathrm{~min}$ at $4{ }^{\circ} \mathrm{C}$. The supernatant was then removed without disturbing the pellet and moved to a separate tube, in which an equal volume of $100 \%$ ethanol was added. RNA was then purified from solution using Direct-zol RNA MiniPrep Kit (Zymo, CA, USA), along with the DNAse step, and elute with $20 \mu \mathrm{L}$ nuclease-free water.

qPCR: cDNA was synthesized following RNA extraction using qScript SuperMix protocol (Quantabio). This cDNA was used as the template for iTaq Universal SYBR Green SuperMix (Bio-Rad, CA, USA), and the protocol was followed to perform qPCR on a Bio-Rad CFX96 Real-Time System.

\section{C. elegans motility assay}

Worms were treated with MCOPPB as previously described. At L4 stage, 3-4 wells of worms were collected and pooled into a microcentrifuge and washed three times with M9+0.01\% Triton-X100 (M9T). Washing was done by spinning the worms at $1000 \mathrm{rpm}$ on a tabletop centrifuge for $30 \mathrm{~s}$, removing the supernatant, and adding fresh M9T. Following the last wash, a p200 pipette tip, with the end cut off, was used to drop $15 \mu \mathrm{L}$ of $30-40$ worms into the center of a NGM plate without food. Using WormLab 
A
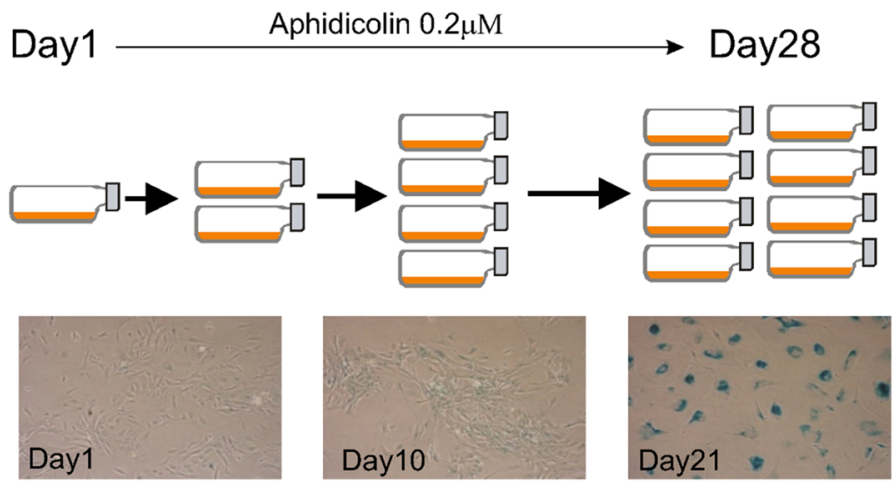

B

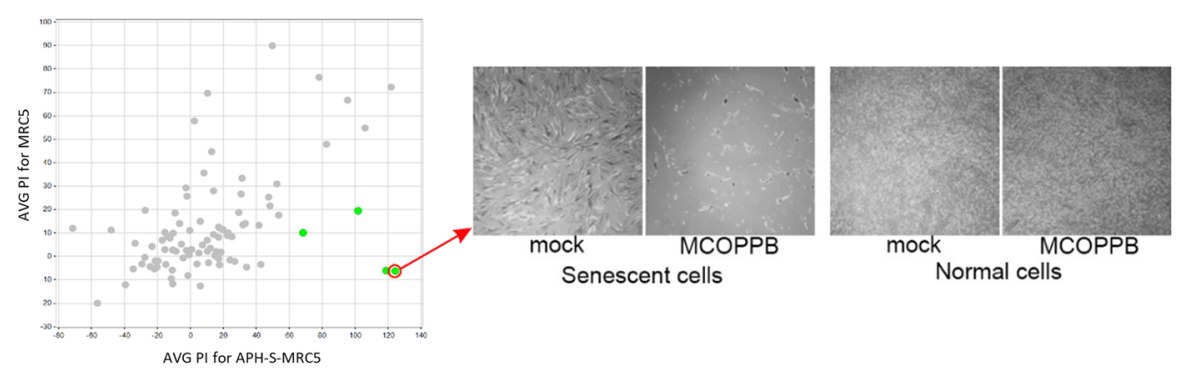

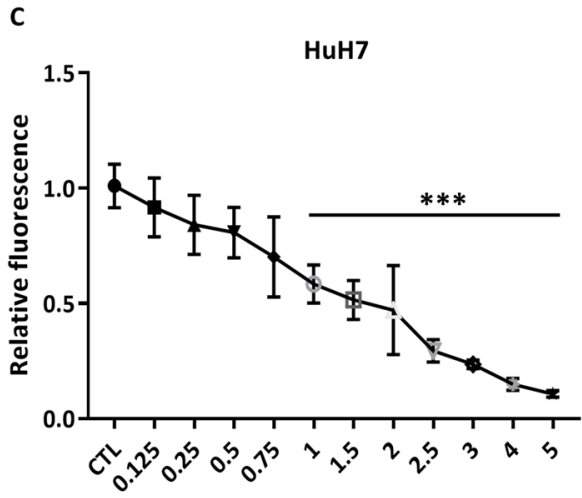

MCOPPB concentration (UM)

D

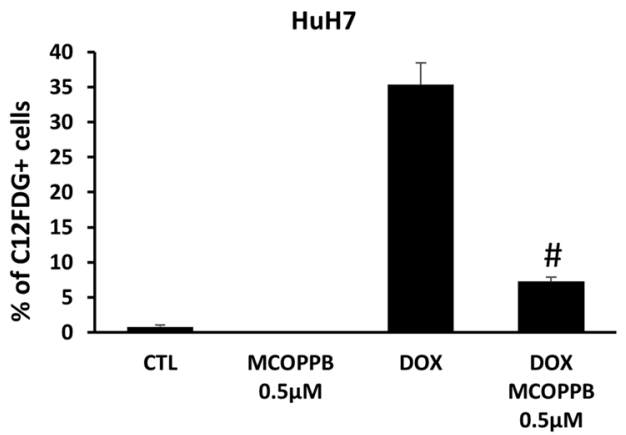

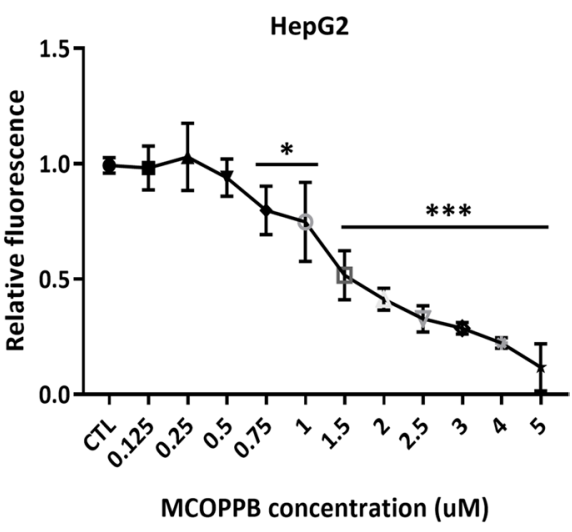

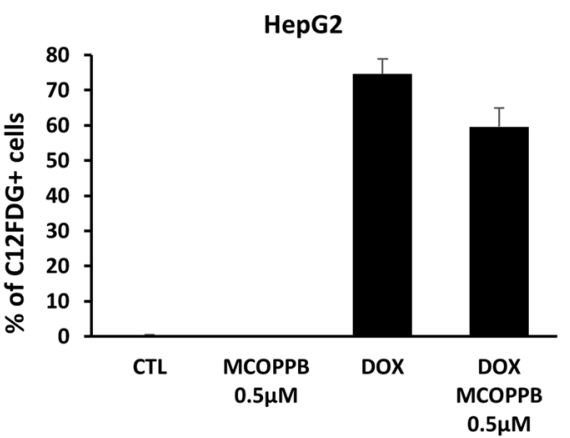


4Fig. 1 Screening for senolytic compounds. A Setup for obtaining a large quantity of senescent MRC5 cells via prolonged exposition to low dose $(0.2 \mu \mathrm{M})$ of replication stress inducer — aphidicolin. The images below are depicting the evolution of SA- $\beta$-gal staining (blue signal) during the treatment procedure. B Screening performed with LOPAC®Pfizer library on MRC5 cells expressed in the dot chart depicting the toxicity (percentage of inhibition, PI) of individual compounds towards normal proliferating cells (y-axis) and senescent cells (x-axis). The chart is accompanied by visual microscopic validation of the strongest hit MCOPPB. C Dose-response of MCOPPB on cell viability. Cells were incubated for $24 \mathrm{~h}$ with increasing concentrations $(0-125-250-500-750 \mathrm{nM}, 1-1.5-2-2.5-3-$ $4-5 \mu \mathrm{M})$ of the drug, before viability assay $(N=6)$. Results are presented as mean \pm SD. D CTL; MCOPPB; DOX; and DOX + MCOOPB-treated HepG2 or Huh-7 cells were incubated with $\mathrm{C} 12 \mathrm{FDG}(N=3)$, and positive cells were detected by flow cytometer. Results are presented as mean \pm SEM. $* p<0.05$ compared to CTL; $* * * p<0.01$ compared to CTL; \# $p<0.05$ compared to DOX

system (MBF Bioscience, VT, USA), a 10-s video was immediately taken of the liquid droplet for the thrashing assay. The liquid was then absorbed by the plate, and worms were allowed to recover on plate for $30 \mathrm{~min}$, before a 30-s video was taken for the crawling assay. Analysis was done using the WormLab software.

\section{Statistical analyses}

Results are expressed as means \pm SEM. Comparisons between groups were performed with the parametric Student's $t$ test or the non-parametric Mann-Whitney $U$ test, as appropriate. The Bonferroni correction was applied in the open-field test analyses in Supplementary Fig. S5. The program used for the analyses was GraphPad Prism Software (version 5.00 for Windows, CA, USA). Asterisks in the figures represent the following: $* p<0.05, \# p<0.05, * * * p<0.01$, and $* * * * p<0.001$.

\section{Results}

High-throughput automatized identification of new senolytics: screening of pharmacologically active compounds on aphidicolin-induced senescent human fibroblasts

We developed and optimized the HTS approach allowing the testing of a relatively large number of chemicals for their potential senolytic activity. We used continuous replication stress induced by low doses of APH, which interferes with ongoing DNA replication, as an efficient inductor of cellular senescence [25]. Replication stress-induced by APH is in multiple aspects similar to activated oncogenes [39] and can generate hard-to-repair and/or irreparable DNA breaks leading to permanent signaling, which inevitably drives cells into senescence [40]. For the screening setup, we used human MRC5 fibroblasts, which were cultivated in the presence of $0.2 \mu \mathrm{M}$ of $\mathrm{APH}$. Cells were regularly divided half to half once they reached the full confluence. The population growth of treated cells gradually slowed down and approximately after 4 weeks stopped completely. By this approach, we were able to obtain a relatively large amount of cells with strong positivity for senescenceassociated beta-galactosidase (SA- $\beta$-gal) staining and all the typical cellular senescence markers, including morphological changes and persistent DNA damage (Fig. 1A and Suppl. Figure 1A). The senescent cells were further seeded in multi-well plates and exposed to the library of pharmacologically active compounds LOPAC®Pfizer, using an automated robotic platform. The toxic response of the senescent cells measured by XTT assay was compared to the parental non-senescent dividing population (HTS data). Four compounds scored as preferentially toxic towards the senescent cells and 1-[1-(1-methylcyclooctyl)4-piperidinyl]-2-[(3R)-3-piperidinyl]-1H-benzimidazole (MCOPPB) molecule scored as the strongest hit (Fig. 1B and Suppl. Figure 1B). Interestingly, MCOPPB is a known potent non-peptide nociceptin/ orphanin FQ peptide (NOP)-receptor full agonist previously shown in preclinical models as a potential anxiolytic [41, 42].

We next tested the senolytic effects of MCOPPB on doxorubicin (DOX)-induced senescent cancer cells, a model of chemotherapy-induced senescence. To this aim, we used two well-established hepatocellular carcinoma (HCC) cell lines (HepG2, Huh-7) [27, 43]. DOX was administered at $100 \mathrm{nM}$ for $24 \mathrm{~h}$ followed by 6 days of washout, as previously described [27, 43], before MCOPPB administration for an additional $24 \mathrm{~h}$. Figure 1C shows the dose-dependent effect of MCOPPB on HepG2 and Huh-7 cell viability, identifying the dose $0.5 \mu \mathrm{M}$ as the maximal cytostatic dose, without displaying cytotoxic effects. To assess the rate of cellular senescence at the end of DOX treatment, we used C12FDG (a fluorogenic substrate 
for $\beta$-gal) for the detection of SA- $\beta$-gal positive cells by flow cytometry. MCOPPB was capable of significantly decreasing up to seven-fold the number of senescent cells in DOX-treated Huh-7 cells (Fig. 1D). A decreasing trend ( $p$ value: 0.096 ) was also observed in DOX-treated HepG2 cells (Fig. 1D). Altogether, these findings show that MCOPPB has cell type-specific senolytic effects in vitro.

\section{MCOPPB reduces locomotion in mice}

The NOP-receptor agonist, MCOPPB, has been characterized pharmacologically to affect emotional and not emotional behavior in vivo at concentrations in the range of $0.1-10 \mathrm{mg} / \mathrm{kg}[41,42]$. We first analyzed the effects of MCOPPB on energy metabolism and behavioral performance in mice thoroughly. The scheme of MCOPPB administration is represented in Fig. 2A. Two groups of mice ( $n=12$, per group) were administered i.p. with MCOPPB ( $5 \mathrm{mg} / \mathrm{kg}$ ) for 5 consecutive days followed by 2 days washout per cycle, for three consecutive cycles (21 days in total) (Fig. 2A). An equal number of control mice were injected with the vehicle. After 21 days, one group of MCOPPB-treated and control mice was processed for PhenoMaster ${ }^{\circledR}$ (Automated Home Cage Phenotyping), and behavioral studies, including open-field test (OFT), elevated plus maze (EPM), Y-maze, and forced swim test (FST) (Group 1). After 21 days, the second group was sacrificed to conduct histology studies (Group 2) (Fig. 2A). After 21 days, three cycles of administration, MCOPPB treatment tended to increase the body weight, although this did not reach statistical significance ( $p=0.09$, Fig. $2 \mathrm{~B})$. We then used PhenoMaster ${ }^{\circledR}$, an automated home cage phenotyping system to monitor mice activity, vital signs, and energy expenditure (calorimetry) (Suppl Fig. 2-3-4). MCOPPB treatment did not affect mice feeding or drinking activities (Suppl. Figure 2A-B), as well as it did not significantly modify the differential distance covered by mice at nighttime (Suppl. Figure 2C) and the number of rearings (counts of interruptions of infrared beams in the Z-dimension) (Suppl. Figure 2D). In terms of physical activity, the number of metabolic equivalents (expressed as $\mathrm{kcal} / \mathrm{h} / \mathrm{kg}$ ) and the energy expenditure (expressed as $\mathrm{kcal} / \mathrm{h}$ ) did not differ between MCOPPB and control mice (Suppl Fig. 3A-B). Similarly, MCOPPB treatment did not affect the respiratory exchange ratio (RER) (Suppl. Figure 3C). Oxygen uptake (VO2) and
A

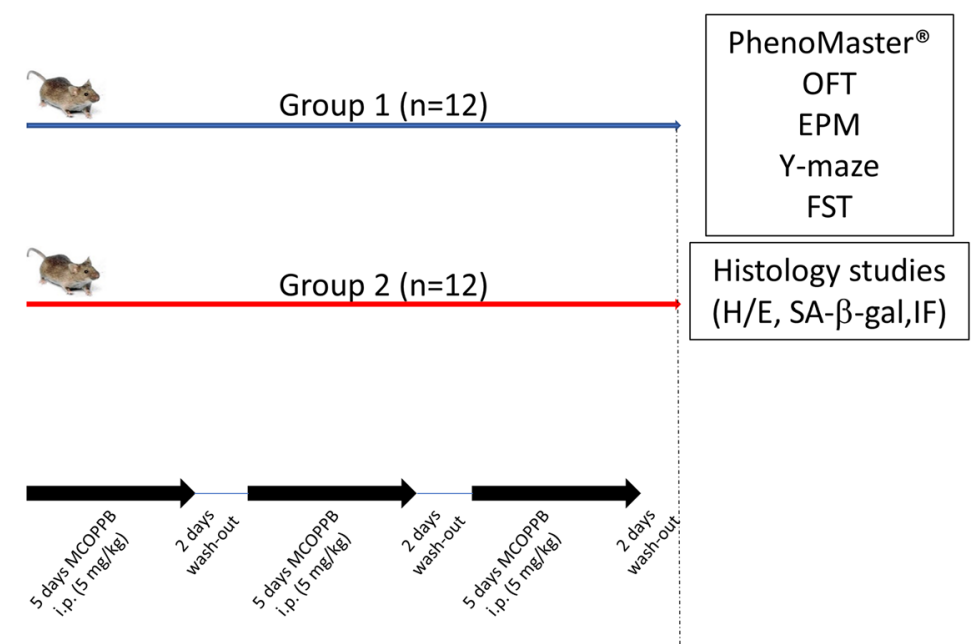

B

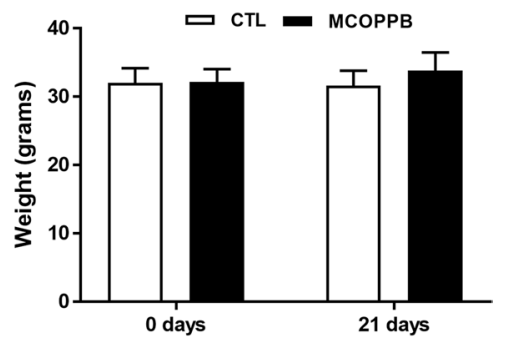

Fig. 2 Scheme of the experimental design of MCOPPB study. A Mice C57JBL6 20 weeks old fed chow diet were treated for 21 days with either $5 \mathrm{mg} / \mathrm{kg}$ of the drug or the vehicle with 2 days of wash-out every 5 days and then were divided into 2 groups $(N=12$ each). Two groups received additional 2 days of wash-out and then were used for the PhenoMaster® experi- ment (Group 1) or sacrificed for Histological analysis at the 28th week (Group 2). B Effects of repeated treatment with MCOPPB or vehicle (CTL) on weight gain. Data are presented as mean \pm SEM of $n=12$ mice/group. * $p<0.05$ vs CTL-treated group 
carbon dioxide production (VCO2) were unchanged, regardless if mice weight was taken into account (Suppl. Figure 4A, B) or not (Suppl. Figure 4C, D).

Behavior is the result of a multitude of physiological and cognitive processes that have to be coordinated and fine-tuned within the organism. Thus, behavioral performance appears to be a suitable readout to test whether MCOPPB induces a detectable behavioral phenotype. MCOPPB-treated mice were subjected to a battery of behavioral tasks for assessing locomotor activity, anxiety-like behavior, learning and memory, and depressive-related behavior. Thus, tests monitoring emotional and non-emotional behavior were performed (Group 1, Fig. 2A). MCOPPB treatment affected the exploratory activity of mice in the open-field test (OFT), as compared to control animals since MCOPPB-treated mice displayed significantly lower ambulatory distance (Fig. 3A), which depended on lower average distance toward the zone border (Fig. 3B) but not toward the zone center (Fig. 3C), where MCOPPBtreated mice spent on average a longer time spent (Fig. 3D). The division of the OFT trials into multiple intervals for analysis is shown in Suppl Fig. 5. In the elevated plus-maze (EPM) test, an exploration-based paradigm for innate anxiety, MCOPPB treatment did not affect the exploration of the aversive open arms, a parameter to assess anxiety-like behavior (Suppl. Figure 6). We then undertook a behavioral test for assessing potential antidepressant compounds (forced swimming test, FST) and a cognitive/memory test for assessing spatial recognition memory (Y-maze test) in mice. Mice treated with MCOPPB showed a significant decrease of mobility time in the FST as index of depressive-like behavior ( $p<0.001$; Fig. 3E). On the other hand, MCOPPB treatment did not affect the cognitive performance in the Y-maze test (Fig. 3F). Overall, treatment with MCOPPB treatment significantly hampered locomotor activity in mice, which could underlie the behavioral response in the FST. Decreased locomotion was not accompanied by changes in energy metabolism/expenditure or by alterations in cognitive performance.

MCOPPB is a potent senolytic and modulates lipid storage in the adipose tissue and the liver

Adipocytes are the major storage site for fat, and this can be accomplished by expanding the available adipose cells. Adipose tissue is the largest organ in
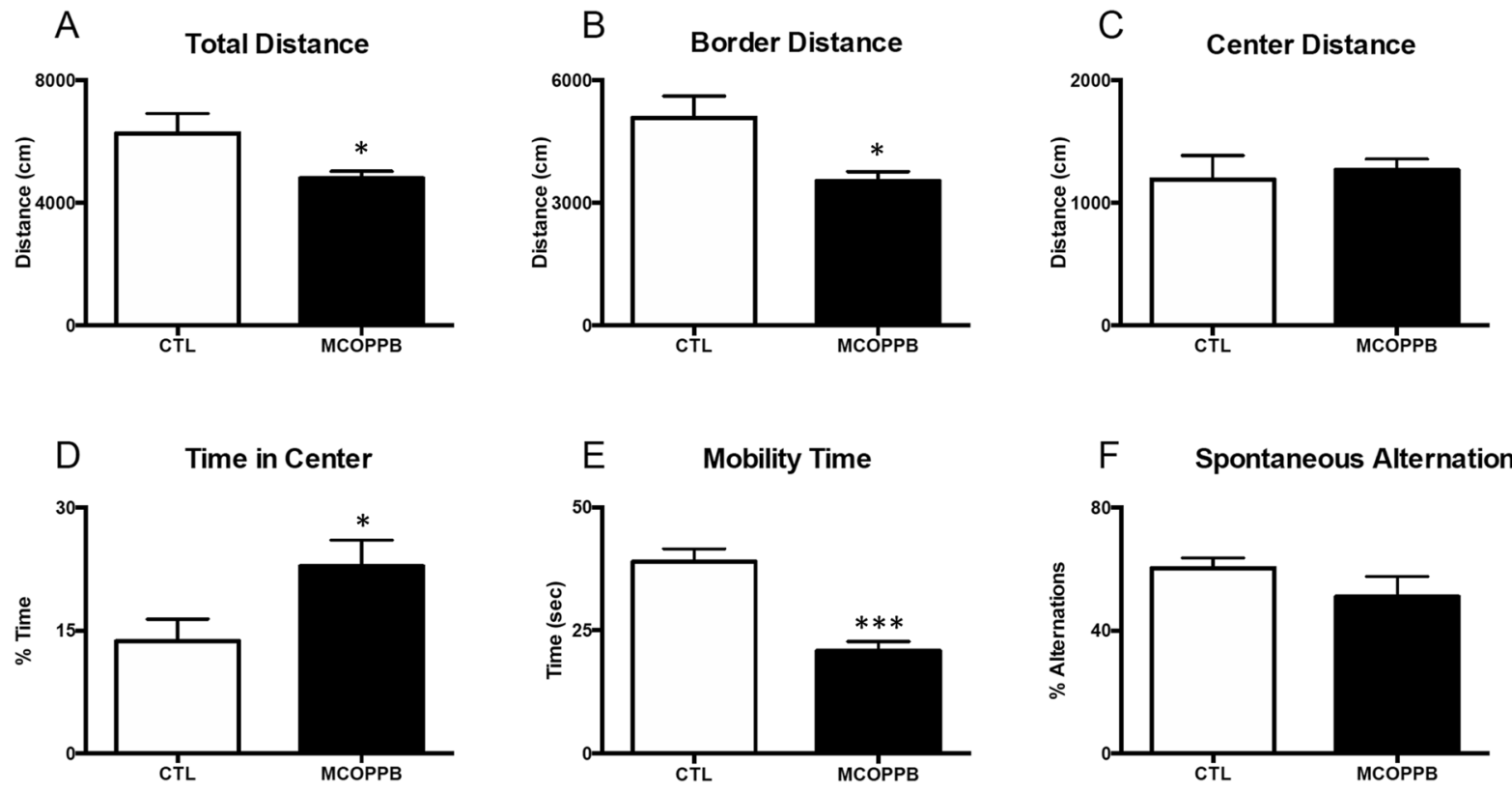

Fig. 3 Effects of repeated treatment with MCOPPB or vehicle (CTL) on A-D locomotor activity in the open-field test (OFT), $\mathbf{E}$ mobility time in the forced swim test (FST), $\mathbf{F}$ cog- nitive performance, and in the Y-maze test of mice. Data are presented as mean \pm SEM of $n=12$ mice/group. ${ }^{*} p<0.05$ and $* * * p<0.01$ vs CTL group 
humans. Senescent cells accumulate in adipose tissue of obese humans and rodents and have a direct pathogenic role in the development of metabolic dysfunction $[44,45]$. SA- $\beta$-gal histological analyses of visceral white adipose tissues revealed a $\sim 70 \%$ reduction in the number of senescent cells upon MCOPPB treatment (Fig. 4A). However, we detected a 20\% increase in adipocyte size (area) in MCOPPB-treated mice compared to control mice even upon a chow diet, which may be consistent with the decreased locomotion and the trend in increased body weight as described above (Fig. 4B). The liver is yet another major hub for nutrient metabolism and disease- or age-dependent cellular senescence-regulated liver disease progression $[13,46]$. Similarly to the adipose tissue, MCOPPB decreased by $70 \%$ the number of senescent cells in the hepatic parenchyma (Fig. 5A). No significant differences were detected in ballooning and inflammatory foci, as well as in glycogen content, between the two groups (Fig. 5B-C), while increased lipid storage was also evident in the liver where analysis of parenchymal architecture revealed early-stage steatosis (steatosis score 0.6) in MCOOPB-treated mice (Fig. 5C) compared to the control group (steatosis score 0 ). These histological data suggest that the

A

SA- $\beta$-Gal staining

CTL
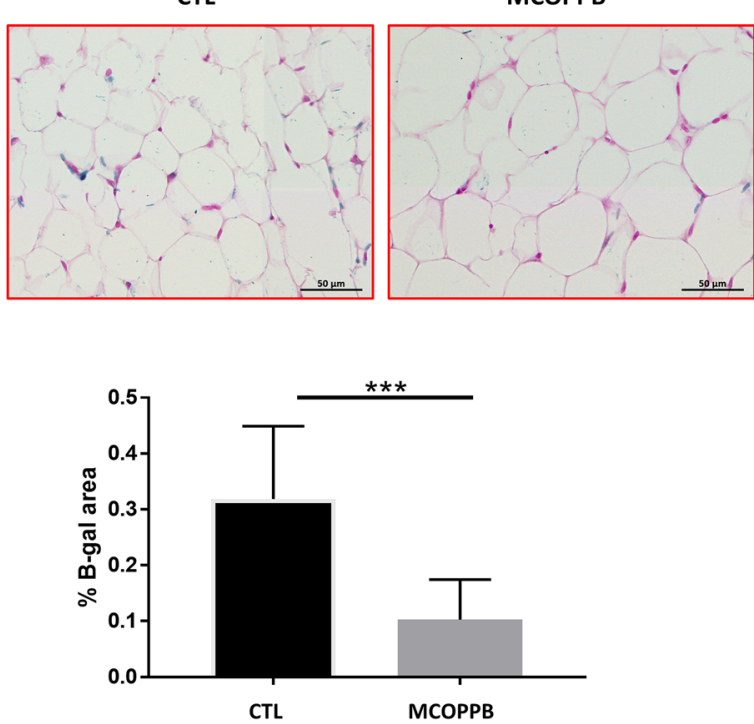

Fig. 4 MCOPPB decreases cellular senescence and increases fat accumulation in WAT adipocytes. A Representative SA- $\beta$ Gal staining and $\mathbf{B}$ representative $H \& E$ images of white adipose tissue from control and MCOPPB treated mice. The
MCOPPB-dependent clearance of senescence cells is associated with mild hepatic stress, which might be explained by the decreased locomotion and the tendency towards weight gain as non-senolytic side effects of MCOPPB treatment.

To unravel the gene expression patterns and related signaling pathways that might be responsible for the observed changes upon MCOPPB treatment, we analyzed whole livers from control and MCOPPB-treated mice by RNA-Seq $(n=3-5$ per group). Treatment with MCOPPB had a substantial effect on the overall gene expression profile (Fig. 6A). Principal component analysis (PCA) identified a clear clustering of samples according to the experimental group (Fig. 6B). Figure 6C displays the main modulated pathways and the differential expressed genes belonging therein, identified using an absolute $z$-score value above 1.7 as a threshold and a $p$ value $<0.0001$, between MCOPPB and the control group. Specifically, these processes are involved in the immune response to pathogens, TREM1 signaling, mTOR signaling, hepatic stellate activation, and cellular senescence (Fig. 6C). Referred to the latter, the RNA-Seq showed a slightly higher GLB1 mRNA expression in the liver of MCOPPB-treated animals

B

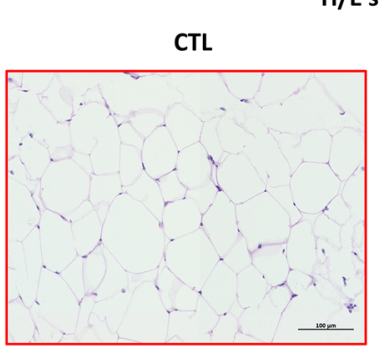

H/E staining
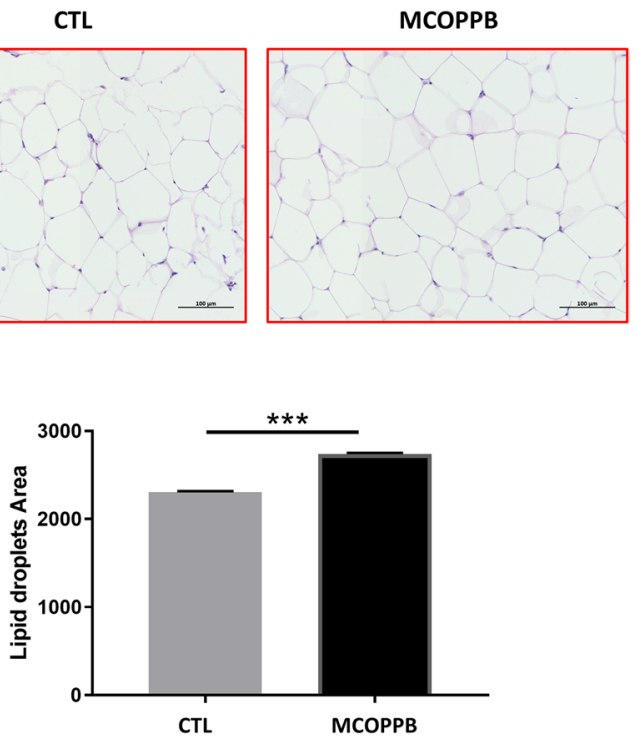

SA- $\beta$-Gal positive and lipid droplets area were calculated in 10 random high-power fields $(\mathrm{HPF})$ at $200 \times$ magnification and expressed as means. $* * * p<0.01$; compared to CTL 


\section{A}
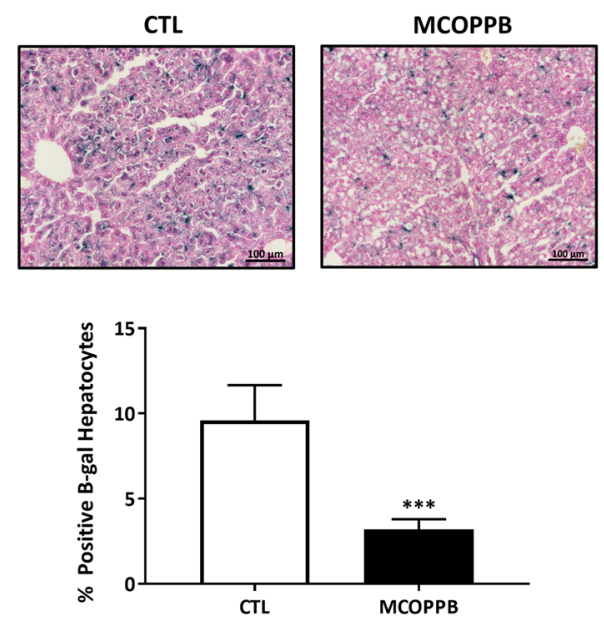

B
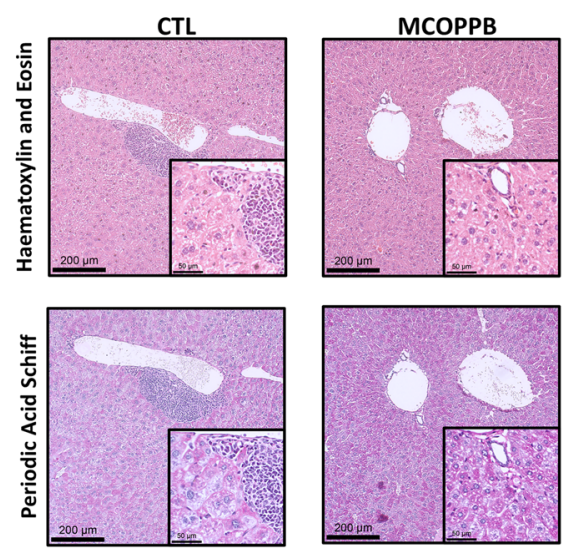

C

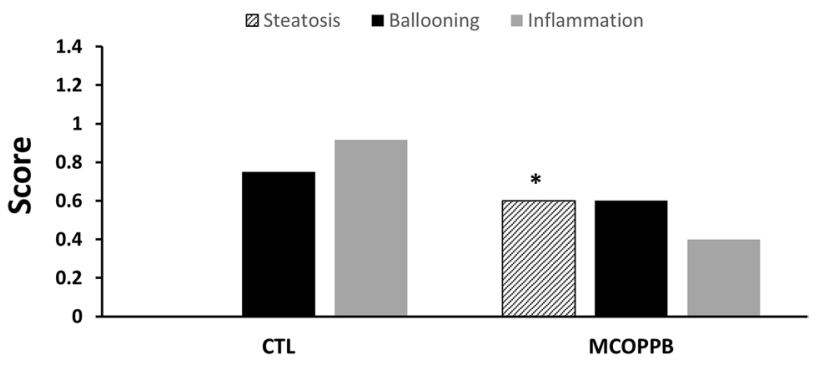

Fig. 5 MCOOPB decreases cellular senescence in mice livers. A Representative SA- $\beta$-Gal staining images from livers of control and MCOPPB treated mice. The SA- $\beta$-Gal positive areas were calculated in 10 random high-power fields (HPF) at $200 \times$ magnification and expressed as means. MCOOPB induces mild hepatic stress in mice. B Representative liver
$\mathrm{H} \& \mathrm{E}$ and PAS images from CTL and MCOPPB treated mice. C Steatosis, ballooning, and inflammation score of CTL and MCOPPB groups. The steatosis score for CTL group is zero. ${ }^{*} p<0.05$; compared to CTL. $* * * p<0.01$; compared to CTL $(\log 2 \mathrm{FC}=0.73 ; p$ value $=0.009)$, suggesting that the lower MCOPPB-dependent SA- $\beta$-gal activity in the liver is not due to lower mRNA expression. The genes enriched in the pathways represented in Fig. 6C were further plotted using STRING pathway analysis, uncovering Toll-like receptor 4 (TLR4) as a nodal hyper-connected hub (Fig. 6D). TLR4 constitutes a fundamental signaling system in modulating the function of macrophages, which can initiate an inflammatory response with the production of cytokines [47].

Moreover, we compared our liver RNA-Seq data with three further publically available genome-wide expression profiles in young and old mouse liver, showing that the MCOPPB-treated group has similarities with the young groups, with 84 genes being the most discriminating between groups (Suppl. Figure 7A) and the most similar intra-groups. The CYP families of genes and, in particular, CYP450 represented the main involved genes, as displayed in the STRING network (Suppl. Figure 7B).

Hepatic macrophages play a central role in maintaining homeostasis in the liver, as well as in the initiation and progression of liver diseases. Macrophages share several similarities and interplay with senescent cells [48]. Both cell types display phagocytosis, lysosomal expansion (with intense SA- $\beta$-gal staining), metabolic reprogramming, a secretory phenotype, and cell cycle arrest. To assess the impact of MCOPPB on the hepatic macrophage population in mice, we performed liver immunofluorescence staining with F4/80 receptor, highly and constitutively expressed in this cell type, and for $\beta$-gal. MCOPPB treatment led to a decrease in the number of $\mathrm{F} 4 / 80$ positive, $\beta$-gal positive cells, and double-positive senescent macrophages in the liver parenchyma (Fig. 7A-E), reflecting shrinkage of the hepatic macrophage population. 
Fig. 6 MCOOPB regulates senescence, immune, and pro-oxidative pathways in mice liver. A Heatmap showing differences in mRNA expression levels, B principal component analysis of the compared groups showing samples clustering on the first two principal components, $\mathbf{C}$ Bar plot reporting $Z$-score of biological pathways in crescent statistical significance order (bar length), and D STRING diagram displaying the protein interaction among the more representative pathways between control and MCOPPB-treated mice ( $n=3-5$ per group)

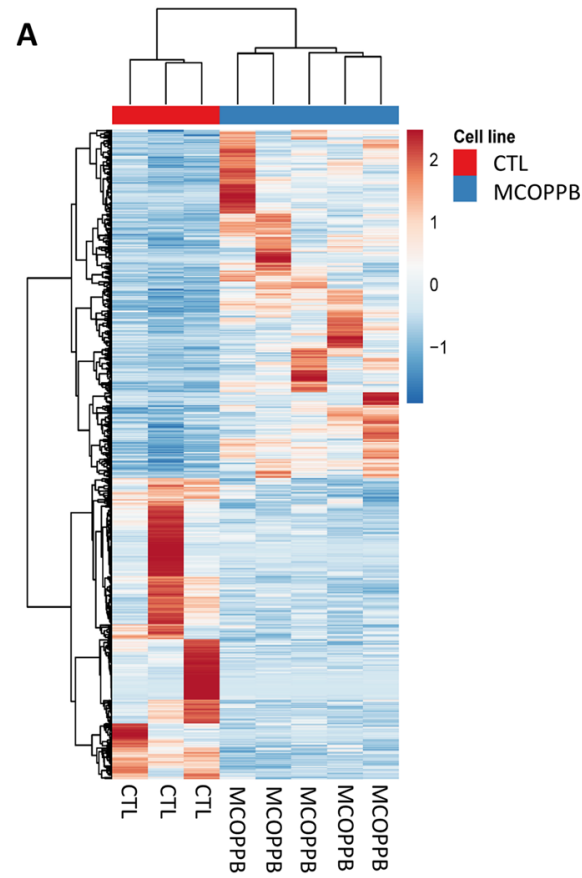

B

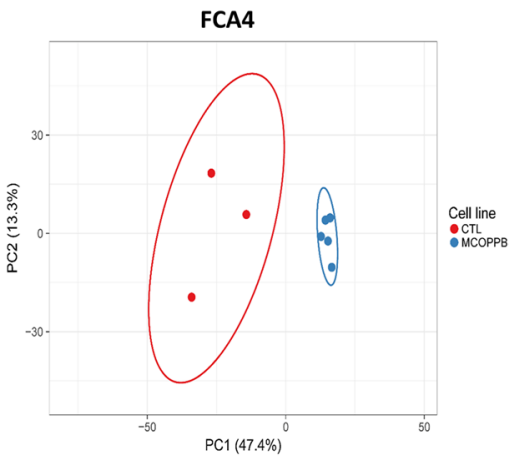

D

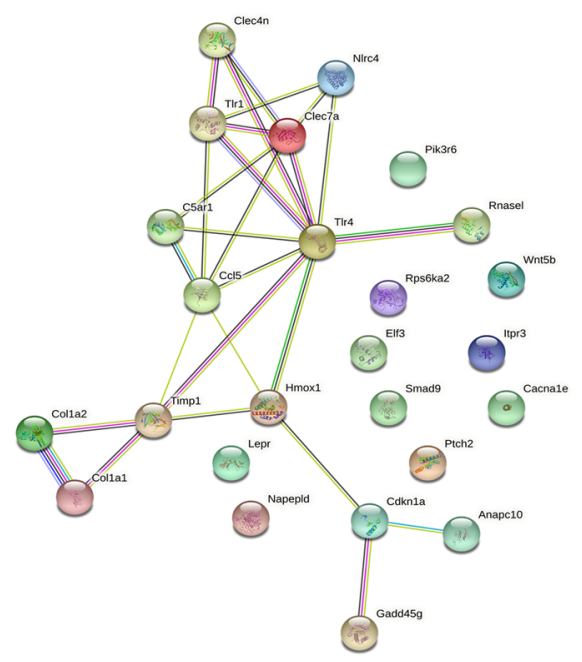


MCOPPB induces lipid accumulation and affects dod-24 gene expression in the nematode Caenorhabditis elegans

To study the potential evolutionarily conserved effects of MCOPPB treatment, we turned to the invertebrate model organism $C$. elegans. The average lifespan of the worm is about 2-3 weeks; they have a transparent body, making it easy to visualize lipid storage in whole animals; and although $C$. elegans and mammals are evolutionarily distant, they share many homologous genes making them an effective system for studying longevity and metabolic functions. Using a liquid culture drug delivery system [49], we exposed worms to vehicle control or MCOPPB and measured lipid abundance using Nile red staining at larval stage 4 (L4) [50]. We found that worms exposed to MCOPPB had increased levels of lipid storage at this stage in life, compared to their untreated counterparts (Fig. 8A). Because of the increase in lipid storage following MCOPPB treatment in mice, we were interested in the effects the drug may have on longevity and motility. We measured several metrics of movement in animals that were swimming/thrashing [51] in liquid or crawling on a NGM agar plate. Total thrashing activity is the measure of how much area the worm covers when making a stroking motion, which is then normalized to the amount of time it takes to make two strokes. MCOPPB treatment decreased the level of thrashing activity a worm performs while swimming in a liquid drop (Fig. 8B). We did not find any differences in motility from crawling worms treated with MCOPPB. Surprisingly, despite changes in these physiological parameters, the lifespan of worms treated with MCOPPB, was indistinguishable from worms treated with vehicle control (Fig. 8C).

The insulin signaling pathway is an essential regulatory pathway in $C$. elegans that is involved in energy homeostasis, lipid metabolism, and longevity [52-54]. DAF-16 is a widely studied transcription factor (the sole ortholog of FOXO family in mammals) that is regulated by insulin signaling, is responsible for the regulation of many downstream metabolism genes, and is necessary for many lifespan extension treatments $[55,56]$. Because of the varied results on lipid storage and longevity, we sought to determine how MCOPPB treatment might affect the expression of genes that have been implicated in longevity and that are regulated by DAF-16, by qPCR analysis. Genes associated with pro-longevity and regulated by DAF-16 (ctl-2, prdx-3, and $m t l-1)$ did
A

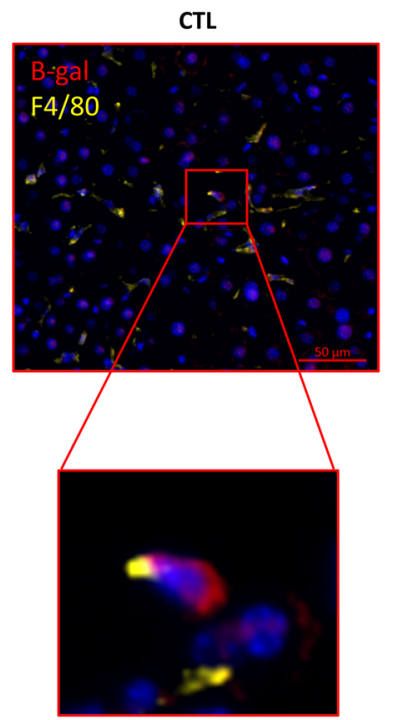

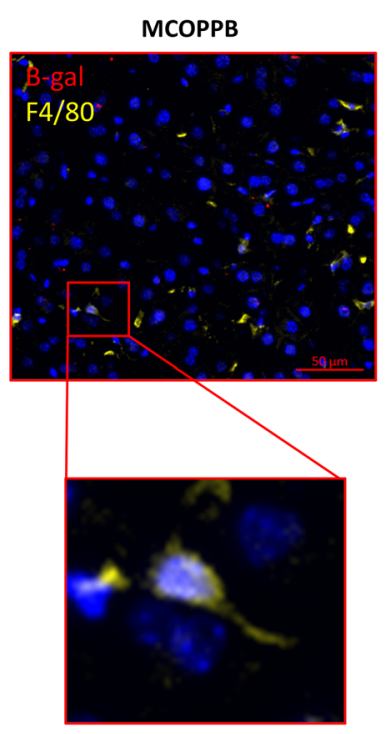

Fig. 7 MCOPPB decreases the number of macrophages and senescent cells in the mouse liver. A Immunofluorescence images of F4-80/B-gal double-positive cells. Frequency of
B
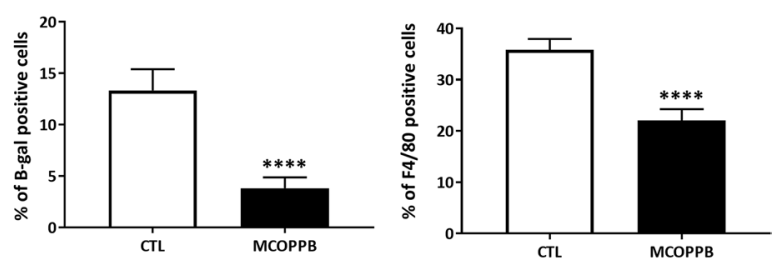

D

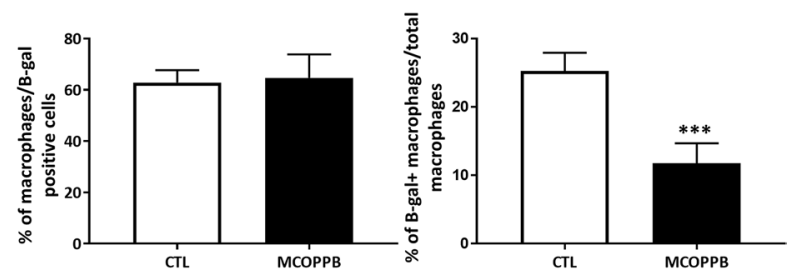

B positive cells for F4-80, C positive cells for B-gal, D macrophages over B-gal+cells, and $\mathbf{E} \mathbf{B}$-gal + macrophages over total macrophages. Indicated in $\%, * * * p<0.01, * * * * p<0.0001$ 
A

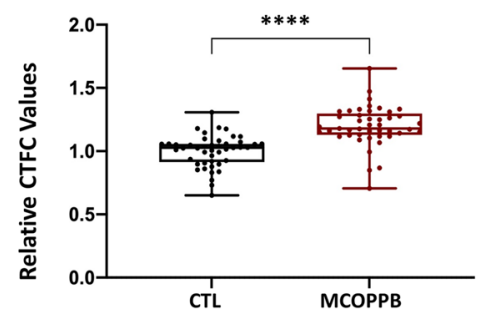

C

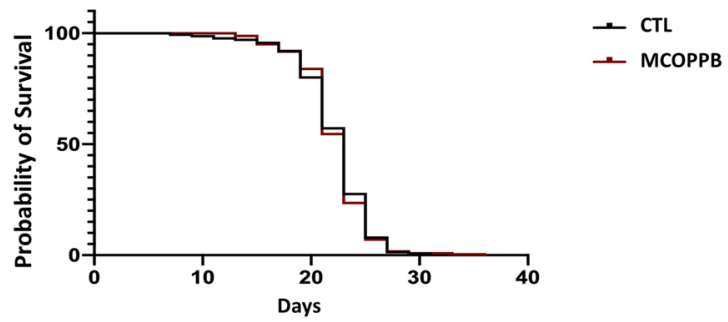

Fig. 8 MCOPPB treatment increases lipid levels but decreases motility and is inconsequential to lifespan in C. elegans. A Quantification of lipid content in worms at L4 stage stained

not have any significant changes in expression, which is consistent with our lifespan data (Fig. 8D). Intriguingly, dod-24, a transcript regulated by both DAF-16 [57] and SKN-1 [58] showed a significant increase following MCOPPB treatment. dod-24 has been implicated with stress and pathogen responses [58] (Fig. 8D).

\section{Discussion}

Senescent cell clearance is believed to induce rejuvenation and longevity. In animal models, senolytic drugs have been shown to delay several age-associated disorders, improve physical and cognitive function, and extend lifespan [59, 60]. Because known senolytic drugs have limited diversity for their modeof-action and the effect changes in a cell-type-specific manner [61], new and effective senolytics drugs are needed before translating them to a medical setting.

In this study, we identified the NOP agonist and anxiolytic agent 1-[1-(1-methylcyclooctyl)-4piperidinyl]-2-[(3R)-3-piperidinyl]-1H-benzimi-

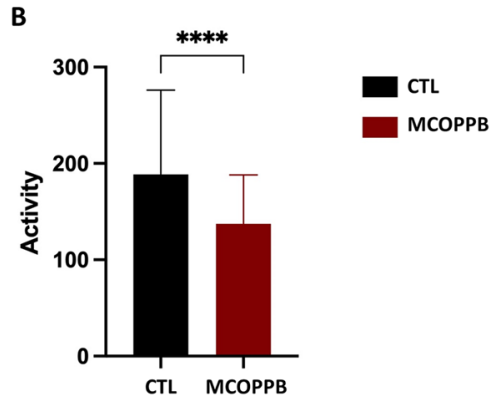

D

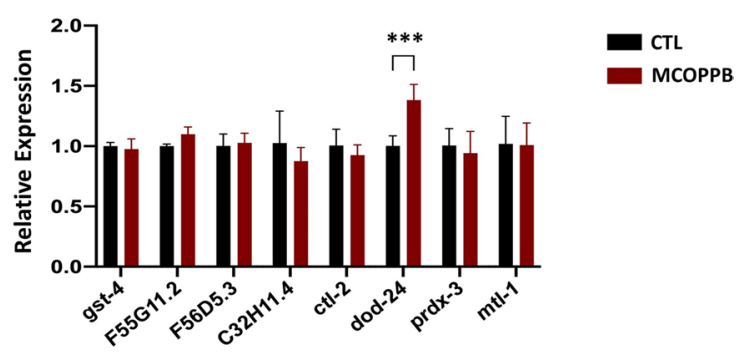

with Nile red, B activity measurement, C lifespan, and D qPCR of SKN-1 and DAF-16 target genes of worms treated with control or MCOPPB. ${ }^{* * *} p<0.01, * * * * p<0.0001$

dazole (MCOPPB) [41, 42, 62], as a new senolytic agent, in vitro and in vivo. While confirming its anxiolytic effects, accompanied by a transient decrease in locomotor activity and by an increase of senescent cells specifically in the motor cortex, the compound showed a decrease in the senescence cell burden in the liver and fat tissue. This effect was accompanied by mild liver stress, characterized by a low grade of steatosis, and by an increase in the adipocytes diameter. We attribute the increased adiposity to the reduced locomotion and the slight increase in body weight observed upon MCOPPB treatment. No structural changes were observed in the other tissues analyzed. It could be possible that a wide-time interval dose could be a better approach to avoid the hepatic side effects.

Studies on known senolytics have highlighted a cell type-dependent effect. Zhu et al. showed that D alone, preferentially reduced viability and caused cell death of senescent human preadipocytes, but was much less effective on senescent HUVECs [14], while on the contrary Q reduced senescent HUVECs, but was less effective on preadipocytes [14]. Moreover, 
yet another senolytic ATB263 adversely affects the viability of various types of senescent cells such as human lung fibroblasts (IMR-90), human umbilical vein endothelial cells (HUVECs), and MEFs, but not of human senescent primary preadipocytes [63]. It has recently been demonstrated that the senolytic piperlongumine preferentially kills senescent human WI-38 fibroblasts when senescence is induced by ionizing radiation, replicative exhaustion, or ectopic expression of the Ras oncogene [64]. Both compounds ATB263 and piperlongumine were much less effective, or not at all, on replicative exhaustion senescence [64, 65].

In this study, MCOPPB was effective in killing APH-induced senescent fibroblasts and the doxorubicin-induced senescent Huh-7 cells, while it showed only a trend in reducing doxorubicin-senescent HepG2 cells. MCOPPB is the most potent novel non-peptide NOP full agonist drug in vitro [62]. One of the earliest therapeutic indications for MCOPPB and other NOP agonists was against anxiety disorders, with a different profile as compared to benzodiazepines. Indeed, Jenck et al. first reported the anxiolytic-like effects of intracerebroventricular treatment with N/OFQ at low nonsedating doses in several behavioral paradigms for assessing anxiety-like behavior in rodents [66]. Soon after, the same group demonstrated that the NOP full agonist Ro 64-6198 elicited anxiolytic-like effects, in different models for assessing distinct types of anxiety states (i.e., spontaneous and conditioned anxiety-like behavior) in rats [67]. Numerous studies documented that anxiety is a risk factor for many age-related medical conditions, such as coronary heart disease, diabetes, and disability, as well as for global mortality [68]. However, preclinical studies suggested that anxiolytics may normalize some hallmarks of accelerated aging and exert a neuroprotective effect. In mice, alprazolam, zolpidem, and buspirone ameliorated the oxidant/ antioxidant balance decreasing nitrite concentration and lipid peroxidation in the brain [68], and the SSRI fluoxetine reversed the decreased activity of telomerase in the hippocampus induced by chronic mild stress [69]. Moreover, a recent report showed that pharmacogenetic and pharmacologic (using D+Q) clearance of senescent cells alleviated obesity-related anxiety and fatty liver in mice [70].

NOP agonists, upon systemic administration, show significant antinociceptive efficacy comparable to morphine [71]. The N/OFQ peptide precursor (ppN/ OFQ) and the NOP receptor are widely expressed in the nervous system as well as in peripheral organs and immune cells [72]. A growing body of evidence suggests a complex role for N/OFQ-NOP receptor system in modulating immune functions, acting either as a stimulator or an inhibitor depending on the nature of the stimulus, N/OFQ concentrations, and timing of NOP activation, besides the sites of immune activation [72]. The immune system plays a fundamental role in senescent cell clearance. Clearance of senescent cells is driven by CD4( + ) T cells and monocytes/ macrophages through a process known as senescence surveillance [73]. Our liver RNA-Seq and bioinformatics analysis identified TLRs — notably TLR4 most abundantly expressed on macrophages, as a key transcriptional node. In fact, the total population of liver resident $\mathrm{F} 4 / 80$ and $\beta$-gal positive macrophages was significantly depleted by MCOPPB treatment in mice. Based on the RNA-Seq data, the lower SA-BGal activity in the livers of MCOPPB-treated animals seems not to be due to lower GLB1 mRNA expression but to a lower number of cells displaying activity of SA- $\beta$-galactosidase. The decrease in liver resident macrophages in the liver during infectious and non-infectious stimuli might prime this organ for repair and regeneration [74, 75]. Indeed, macrophages are powerful scavengers and are the major cell type involved in phagocytosis of apoptotic cells, but the fate of macrophages once they have ingested apoptotic cells is open to much debate. There is good evidence that the macrophages, unlike both the neutrophils and the un-activated monocytes, are longlived cells $[76,77]$. This suggests that local apoptosis may not be the immediate fate for these cells, which instead seem to migrate from the inflamed site into close draining lymph nodes [78, 79]. Moreover, even Kupffer cells, long believed to be tissue-fixed macrophages, have been shown to migrate along sinusoid walls with or against blood flow rather than into the lymphatics [80].

Macrophages are key cells in the induction and maintenance of inflammation that characterizes aging [81]. Inflammation and macrophage aging occur in association with immunosenescence, whose hallmark is a reduced efficiency of immunity cells to cope with stressors during aging [82]. We can speculate that the decrease in total and senescent macrophages number in the liver, caused by MCOPPB administration, 
counteract the immunosenescence renewing the macrophage population and reducing the inflammatory processes.

Despite functional and phenotypical similarities between senescent cells and macrophages, the interplay between these cell types upon senolytic therapy warrants further investigation.

C. elegans have become a popular animal model to study how drugs may affect lifespan and healthspan, and it has even been used to discover conserved pathways involved in opioid responses [83, 84]. To that end, we decided to use the worm system to study the effects of MCOPPB. Although lifespan was not significantly affected by MCOPPB treatment, several changes to established healthspan parameters were observed. Similar to the mammalian system tested here, treatment with MCOPPB led to an increase in lipid storage and reduced swimming (thrashing in liquid). The effects of MCOPPB on both lipids and motility suggest that MCOPPB may have an evolutionarily conserved role in promoting specific aspects of animal physiology. While lifespan was not significantly affected by MCOPPB treatment, we did see an increase in the expression of $d o d-24$, a DAF-16 controlled gene. $d o d-24$ is not a pro-longevity gene, but it has been implicated with pathogen responses in $C$. elegans [58], suggesting that MCOPPB could have some role in modulating the immune system, consistent with the mice data on TLR4 and macrophages.

Here, we observed a reduction in cell senescence burden in liver and adipose tissue of the MCOPPBtreated mice, accompanied by a mild increase in adiposity. In one study, administration of N/OFQ produced an increase in body weight in mice fed with regular chow or moderately high-fat diet [85], while in a pair-fed experiment where the mice treated with N/OFQ, no increase in body weight was observed, despite was still present an increase of white adipose tissue mass [86]. According to this finding, in our study MCOPPB led to a significant increase in adipocytes size. Similarly, MCOPPB treatment increased overall $C$. elegans organismal lipid density within intestinal cells, which are the major site of lipid storage [38, 87].

There are some limitations in our study. First, although we clearly demonstrated the senolytic effect of MCOPPB in vitro and in vivo, the drug showed side effects including weight gain and hepatic stress: the potential link with the senolytic effects or other actions of the drug has not been assessed. Future studies on the drug mechanism can elucidate the correlations underlying these unwanted effects. Second, in this study, we showed the senolytic effects of MCOPPB on mice after 2 months of treatments, but further experiments with a prolonged administration of the drug would be necessary to evaluate the balance between beneficial and side effects. Moreover, studies on topical application of senolytics have shown to suppress the aging-related skin hyperplasia [88] and improve hair follicle stem cells regeneration [89], while a local administration in an intervertebral disc degeneration murine model resulted in a restored intervertebral disc structure [90]. This suggests that a local, tissue-specific, application could represent a valid alternative in order to avoid systemic detrimental effects. Third, despite the C. elegans is a common model used to assess the effects of drugs on lifespan, the presence of a human or murine ortholog nociceptin receptor in this worm has not been demonstrated. Nevertheless, a recent study suggested that C. elegans has an endogenous opioid system that acts through NPR-17, a peptide with $28 \%$ of identity to human nociceptin [83]. The understanding of the coordination of the MCOPPB-dependent central and peripheral effects in determining nutrient metabolism, energy expenditure, and the turnover of senescent cells warrants further studies.

In conclusion, we describe the powerful senolytic effects of the anxiolytic MCOPPB in peripheral tissues, where it may modulate lipid storing and immune functions. Our study contributes towards the understanding of the complex physiological integration between N/OFQ-NOP receptor signaling, behavioral changes, immune responses, and cellular senescence.

Acknowledgements The authors thank the members of the Center for Translational Medicine (CTM, ICRC) for support. The behavioral experiments were performed by the Preclinical Phenotyping Facility at Vienna BioCenter Core Facilities (VBCF), a member of the Vienna BioCenter (VBC), Austria. The VBCF Preclinical Phenotyping Facility acknowledges funding from the Austrian Federal Ministry of Education, Science \& Research, and the City of Vienna

Author contribution $\mathrm{MM}$ and MV conceptualized the study. MR, KK, TB, DC, ML, MM, MH, and VM designed the methodology. MR, KK, TB, OLR, JF, SG, JDN, AGG, JO, MI, LL, $\mathrm{ML}, \mathrm{PD}$, and SoG performed the experiments and conducted analyses. MR and MV wrote the original draft.

MR, KK, TB, OLR, JF, SG, JDN, AGG, DC, JO, MI, ABT, LL, MF, MM, ML, PD, SoG, MH, JB, TM, VM, SPC, and MV 
reviewed and edited the manuscript. Authors discussed the results and commented on the manuscript. $\mathrm{DC}, \mathrm{ABT}, \mathrm{MF}, \mathrm{MH}$, $\mathrm{TM}, \mathrm{JB}, \mathrm{SPC}$, and MV provided overall supervision.

Funding This work was supported by the European Social Fund and European Regional Development Fund, Project MAGNET (No. CZ.02.1.01/0.0/0.0/15_003/0000492, to M.V.) and Project ENOCH (No. CZ.02.1.01/0.0/0.0/16_019/0000868, to PD, MM, MH, SoG), by the Ministry of Health of the Czech Republic (No. NV18-03-00058 to MV), by the European Commission Horizon 2020 Framework Program (Project 856871TRANSTEM) (ABT, MV), by the National Institute of Aging (NIA) (Research and Development Core at the USC-Buck Nathan Shock Center, P30AG068345, and R01AG058610 to SPC), and by the Czech Ministry of Education, Youth and Sports (CZ-OPENSCREEN- LM2018130, EATRIS-CZ LM2018133 to PD, SoG, MH).

Data availability All the data supporting the findings of this study are available within the article and its Supplementary materials files or from the corresponding author upon reasonable request.

\section{Declarations}

Competing interests $\mathrm{MM}, \mathrm{JB}$, and $\mathrm{MH}$ are co-inventors on a patent application: EP 3552605 A1, utilizing MCOPPB as a medicament with senolytic effects. Other authors declare no other competing interests.

\section{References}

1. Campisi J. Aging, cellular senescence, and cancer. Annu Rev Physiol. 2013. https://doi.org/10.1146/annurev-physi ol-030212-183653.

2. Hodes RJ, et al. Disease drivers of aging. Ann N Y Acad Sci. 2016. https://doi.org/10.1111/nyas.13299.

3. Inouye SK, et al. Geriatric syndromes: clinical, research, and policy implications of a core geriatric concept. J Am Geriatr Soc. 2007. https://doi.org/10.1111/j.1532-5415. 2007.01156.x.

4. McHugh D, Gil J. Senescence and aging: causes, consequences, and therapeutic avenues. J Cell Biol. 2018. https://doi.org/10.1083/jcb.201708092.

5. Childs BG, et al. Senescent cells: an emerging target for diseases of ageing. Nat Rev Drug Discov. 2017. https:// doi.org/10.1038/nrd.2017.116.

6. Schafer MJ, et al. The senescence-associated secretome as an indicator of age and medical risk. JCI Insight. 2020. https://doi.org/10.1172/jci.insight.133668.

7. Gorgoulis V, et al. Cellular senescence: defining a path forward. Cell. 2019. https://doi.org/10.1016/j.cell.2019. 10.005 .

8. Bartek J, Bartkova J, Lukas J. DNA damage signalling guards against activated oncogenes and tumour progression. Oncogene. 2007. https://doi.org/10.1038/sj.onc. 1210881.
9. Pignolo RJ, et al. Reducing senescent cell burden in aging and disease. Trends Mol Med. 2020. https://doi. org/10.1016/j.molmed.2020.03.005.

10. Kirkland JL, et al. The clinical potential of senolytic drugs. J Am Geriatr Soc. 2017. https://doi.org/10.1111/ jgs. 14969.

11. Kirkland JL, Tchkonia T. Cellular senescence: a translational perspective. EBioMedicine. 2017. https://doi.org/ 10.1016/j.ebiom.2017.04.013.

12. Calimport SRG, et al. To help aging populations, classify organismal senescence. Science. 2019. https://doi. org/10.1126/science.aay7319.

13. Ogrodnik $\mathbf{M}$, et al. Cellular senescence drives agedependent hepatic steatosis. Nat Commun. 2017. https:// doi.org/10.1038/ncomms15691.

14. Zhu Y, et al. The Achilles' heel of senescent cells: from transcriptome to senolytic drugs. Aging Cell. 2015. https://doi.org/10.1111/acel.12344.

15. Volochnyuk DM, et al. Evolution of commercially available compounds for HTS. Drug Discov Today. 2019. https://doi.org/10.1016/j.drudis.2018.10.016.

16. Fuhrmann-Stroissnigg H, et al. Identification of HSP90 inhibitors as a novel class of senolytics. Nat Commun. 2017. https://doi.org/10.1038/s41467-017-00314-z.

17. Wakita M, et al. A BET family protein degrader provokes senolysis by targeting NHEJ and autophagy in senescent cells. Nat Commun. 2020. https://doi.org/10. 1038/s41467-020-15719-6.

18. Cho HJ, et al. Identification of SYK inhibitor, R406 as a novel senolytic agent. Aging (Albany NY). 2020. https://doi.org/10.18632/aging.103135.

19. Ozsvari B, et al. Azithromycin and Roxithromycin define a new family of "senolytic" drugs that target senescent human fibroblasts. Aging (Albany NY). 2018. https://doi.org/10.18632/aging.101633.

20. Bucknall RA, et al. Antiviral effects of aphidicolin, a new antibiotic produced by Cephalosporium aphidicola. Antimicrob Agents Chemother. 1973. https://doi.org/10. 1128/aac.4.3.294.

21. Huberman JA. New views of the biochemistry of eucaryotic DNA replication revealed by aphidicolin, an unusual inhibitor of DNA polymerase alpha. Cell. 1981. https://doi.org/10.1016/0092-8674(81)90426-8.

22. Glover TW, et al. DNA polymerase alpha inhibition by aphidicolin induces gaps and breaks at common fragile sites in human chromosomes. Hum Genet. 1984. https:// doi.org/10.1007/BF00272988.

23. Mazouzi A, et al. A Comprehensive Analysis of the Dynamic Response to Aphidicolin-Mediated Replication Stress Uncovers Targets for ATM and ATMIN. Cell Rep. 2016. https://doi.org/10.1016/j.celrep.2016.03.077.

24. Levenson VV, et al. A combination of genetic suppressor elements produces resistance to drugs inhibiting DNA replication. Somat Cell Mol Genet. 1999. https:// doi.org/10.1023/b:scam.0000007136.49230.b3.

25. Marusyk A, et al. p53 mediates senescence-like arrest induced by chronic replicational stress. Mol Cell Biol. 2007. https://doi.org/10.1128/MCB.01316-06.

26. Novakova $Z$, et al. Cytokine expression and signaling in drug-induced cellular senescence. Oncogene. 2010. https://doi.org/10.1038/onc.2009.318. 
27. Kovacovicova $\mathrm{K}$, et al. Senolytic cocktail dasatinib+quercetin $(\mathrm{D}+\mathrm{Q})$ does not enhance the efficacy of senescence-inducing chemotherapy in liver cancer. Front Oncol. 2018. https://doi.org/10.3389/fonc. 2018.00459.

28. Terzian AL, et al. The dopamine and cannabinoid interaction in the modulation of emotions and cognition: assessing the role of cannabinoid CB1 receptor in neurons expressing dopamine D1 receptors. Front Behav Neurosci. 2011. https://doi.org/10.3389/fnbeh.2011. 00049.

29. Stark T, et al. Peripubertal cannabidiol treatment rescues behavioral and neurochemical abnormalities in the MAM model of schizophrenia. Neuropharmacology. 2019. https://doi.org/10.1016/j.neuropharm.2018.11.035.

30. Pamplona FA, et al. Prolonged fear incubation leads to generalized avoidance behavior in mice. J Psychiatr Res. 2011. https://doi.org/10.1016/j.jpsychires.2010.06.015.

31. Hazane F, et al. Behavioral perturbations after prenatal neurogenesis disturbance in female rat. Neurotox Res. 2009. https://doi.org/10.1007/s12640-009-9035-z.

32. Murgatroyd C, et al. Dynamic DNA methylation programs persistent adverse effects of early-life stress. Nat Neurosci. 2009. https://doi.org/10.1038/nn.2436.

33. Direnberger S, et al. Biocompatibility of a genetically encoded calcium indicator in a transgenic mouse model. Nat Commun. 2012. https://doi.org/10.1038/ncomm s2035.

34. Micale V, Kucerova J, Sulcova A. Leading compounds for the validation of animal models of psychopathology. Cell Tissue Res. 2013. https://doi.org/10.1007/ s00441-013-1692-9.

35. Takeda D, et al. Effect of preoperative chemotherapy on postoperative liver regeneration following hepatic resection as estimated by liver volume. World J Surg Oncol. 2013. https://doi.org/10.1186/1477-7819-11-65.

36. Raffaele M, et al., Senescence-like phenotype in postmitotic cells of mice entering middle age. Aging (Albany NY), 2020. https://doi.org/10.18632/aging.103637.

37. Anders S, Pyl PT, Huber W. HTSeq-a Python framework to work with high-throughput sequencing data. Bioinformatics. 2015. https://doi.org/10.1093/bioinformatics/ btu638.

38. Escorcia W, et al. Quantification of lipid abundance and evaluation of lipid distribution in Caenorhabditis elegans by Nile Red and Oil Red O Staining. J Vis Exp. 2018. https://doi.org/10.3791/57352.

39. Vesela E, et al. Common chemical inductors of replication stress: focus on cell-based studies. Biomolecules. 2017. https://doi.org/10.3390/biom7010019.

40. Rossiello F, et al. Irreparable telomeric DNA damage and persistent DDR signalling as a shared causative mechanism of cellular senescence and ageing. Curr Opin Genet Dev. 2014. https://doi.org/10.1016/j.gde. 2014.06.009.

41. Hirao A, et al. Pharmacological characterization of the newly synthesized nociceptin/orphanin FQ-receptor agonist 1-[1-(1-methylcyclooctyl)-4-piperidinyl]-2[(3R)-3-piperidinyl]-1H-benzimidazole as an anxiolytic agent. J Pharmacol Sci. 2008. https://doi.org/10.1254/ jphs.fp0071742.
42. Holanda VAD, et al. Modulation of the NOP receptor signaling affects resilience to acute stress. J Psychopharmacol. 2019. https://doi.org/10.1177/0269881119 864942.

43. Kovacovicova K, Vinciguerra M. Isolation of senescent cells by iodixanol (OptiPrep) density gradient-based separation. Cell Prolif. 2019. https://doi.org/10.1111/cpr. 12674.

44. Tchkonia T, et al. Fat tissue, aging, and cellular senescence. Aging Cell. 2010. https://doi.org/10.1111/j.14749726.2010.00608.x.

45. Palmer AK, et al. Targeting senescent cells alleviates obesity-induced metabolic dysfunction. Aging Cell. 2019. https://doi.org/10.1111/acel.12950.

46. Sheedfar F, et al. Liver diseases and aging: friends or foes? Aging Cell. 2013. https://doi.org/10.1111/acel. 12128.

47. Miura K, Ishioka M, Iijima K. The roles of the gut microbiota and toll-like receptors in obesity and nonalcoholic fatty liver disease. J Obes Metab Syndr. 2017. https://doi. org/10.7570/jomes.2017.26.2.86.

48. Behmoaras J, Gil J. Similarities and interplay between senescent cells and macrophages. J Cell Biol. 2021. https://doi.org/10.1083/jcb.202010162.

49. Ye X, et al. A pharmacological network for lifespan extension in Caenorhabditis elegans. Aging Cell. 2014. https:// doi.org/10.1111/acel.12163.

50. Nhan JD, Curran SP. Metabolic assessment of lipid abundance and distribution. Methods Mol Biol. 2020. https:// doi.org/10.1007/978-1-0716-0592-9_9.

51. Laranjeiro R, et al. Single swim sessions in C. elegans induce key features of mammalian exercise. BMC Biol. 2017. https://doi.org/10.1186/s12915-017-0368-4.

52. Apfeld J, et al. The AMP-activated protein kinase AAK-2 links energy levels and insulin-like signals to lifespan in C. elegans. Genes Dev. 2004. https://doi.org/10.1101/gad. 1255404.

53. Lee S, Dong HH. FoxO integration of insulin signaling with glucose and lipid metabolism. J Endocrinol. 2017. https://doi.org/10.1530/JOE-17-0002.

54. Jia K, Chen D, Riddle DL. The TOR pathway interacts with the insulin signaling pathway to regulate C. elegans larval development, metabolism and life span. Development. 2004. https://doi.org/10.1242/dev.01255.

55. Lin K, et al. Regulation of the Caenorhabditis elegans longevity protein DAF-16 by insulin/IGF-1 and germline signaling. Nat Genet. 2001. https://doi.org/10.1038/88850.

56. Lee SS, et al. DAF-16 target genes that control C. elegans life-span and metabolism. Science. 2003. https:// doi.org/10.1126/science.1083614.

57. Kaletsky R, et al. The C. elegans adult neuronal IIS/ FOXO transcriptome reveals adult phenotype regulators. Nature. 2016. https://doi.org/10.1038/nature16483.

58. Nhan JD, et al. Redirection of SKN-1 abates the negative metabolic outcomes of a perceived pathogen infection. Proc Natl Acad Sci U S A. 2019. https://doi.org/10. 1073/pnas.1909666116.

59. Xu M, et al. Senolytics improve physical function and increase lifespan in old age. Nat Med. 2018. https://doi. org/10.1038/s41591-018-0092-9. 
60. Baker DJ, et al. Clearance of p16Ink4a-positive senescent cells delays ageing-associated disorders. Nature. 2011. https://doi.org/10.1038/nature10600.

61. Blagosklonny MV. Paradoxes of senolytics. Aging (Albany NY). 2018. https://doi.org/10.18632/aging. 101750.

62. Hayashi S, et al. Novel non-peptide nociceptin/orphanin FQ receptor agonist, 1-[1-(1-Methylcyclooctyl)-4piperidinyl]-2-[(3R)-3-piperidinyl]-1H-benzimidazole: design, synthesis, and structure-activity relationship of oral receptor occupancy in the brain for orally potent antianxiety drug. J Med Chem. 2009. https://doi.org/10. 1021/jm7012979.

63. Zhu Y, et al. Identification of a novel senolytic agent, navitoclax, targeting the Bcl-2 family of anti-apoptotic factors. Aging Cell. 2016. https://doi.org/10.1111/acel.12445.

64. Wang Y, et al. Discovery of piperlongumine as a potential novel lead for the development of senolytic agents. Aging (Albany NY). 2016. https://doi.org/10.18632/ aging. 101100 .

65. Chang J, et al. Clearance of senescent cells by ABT263 rejuvenates aged hematopoietic stem cells in mice. Nat Med. 2016. https://doi.org/10.1038/nm.4010.

66. Jenck F, et al. Orphanin FQ acts as an anxiolytic to attenuate behavioral responses to stress. Proc Natl Acad Sci U S A. 1997. https://doi.org/10.1073/pnas.94.26.14854.

67. Jenck F, et al. A synthetic agonist at the orphanin FQ/ nociceptin receptor ORL1: anxiolytic profile in the rat. Proc Natl Acad Sci U S A. 2000. https://doi.org/10.1073/ pnas.090514397.

68. Perna $\mathrm{G}$, et al. Are anxiety disorders associated with accelerated aging? A focus on neuroprogression Neural Plast. 2016. https://doi.org/10.1155/2016/8457612.

69. Zhou QG, et al. Hippocampal telomerase is involved in the modulation of depressive behaviors. J Neurosci. 2011. https://doi.org/10.1523/JNEUROSCI.0805-11.2011.

70. Ogrodnik M, et al. Obesity-induced cellular senescence drives anxiety and impairs neurogenesis. Cell Metab. 2019. https://doi.org/10.1016/j.cmet.2018.12.008.

71. Zaveri NT, Meyer ME. NOP-targeted nonpeptide ligands. Handb Exp Pharmacol. 2019. https://doi.org/10.1007/ 164_2019_213.

72. Toll L, et al. Nociceptin/orphanin FQ receptor structure, signaling, ligands, functions, and interactions with opioid systems. Pharmacol Rev. 2016. https://doi.org/10.1124/pr. 114.009209.

73. Burton DGA, Stolzing A. Cellular senescence: immunosurveillance and future immunotherapy. Ageing Res Rev. 2018. https://doi.org/10.1016/j.arr.2018.02.001.

74. Li Z, Weinman SA. Regulation of hepatic inflammation via macrophage cell death. Semin Liver Dis. 2018. https:// doi.org/10.1055/s-0038-1670674.

75. Ferreira-Gonzalez $\mathrm{S}$, et al. Cellular senescence in liver disease and regeneration. Semin Liver Dis. 2021. https:// doi.org/10.1055/s-0040-1722262.

76. Mangan DF, Wahl SM. Differential regulation of human monocyte programmed cell death (apoptosis) by chemotactic factors and pro-inflammatory cytokines. J Immunol. 1991.

77. Perera LP, Waldmann TA. Activation of human monocytes induces differential resistance to apoptosis with rapid down regulation of caspase-8/FLICE. Proc Natl Acad Sci U S A. 1998. https://doi.org/10.1073/pnas.95.24. 14308.

78. Bellingan GJ, et al. In vivo fate of the inflammatory macrophage during the resolution of inflammation: inflammatory macrophages do not die locally, but emigrate to the draining lymph nodes. J Immunol. 1996.

79. Harmsen AG, et al. The role of macrophages in particle translocation from lungs to lymph nodes. Science. 1985. https://doi.org/10.1126/science.4071052.

80. MacPhee PJ, Schmidt EE, Groom AC. Evidence for Kupffer cell migration along liver sinusoids, from highresolution in vivo microscopy. Am J Physiol. 1992. https://doi.org/10.1152/ajpgi.1992.263.1.G17.

81. Franceschi $\mathrm{C}$, et al. Inflamm-aging. An evolutionary perspective on immunosenescence. Ann N Y Acad Sci. 2000. https://doi.org/10.1111/j.1749-6632.2000.tb06651.x.

82. Prattichizzo F, et al. Senescence associated macrophages and "macroph-aging": are they pieces of the same puzzle? Aging (Albany NY). 2016. https://doi.org/10.18632/ aging.101133.

83. Cheong MC, et al. An opioid-like system regulating feeding behavior in C. elegans. Elife. 2015. https://doi.org/10. 7554/eLife.06683.

84. Wang D, et al. Genetic behavioral screen identifies an orphan anti-opioid system. Science. 2019. https://doi.org/ 10.1126/science.aau2078.

85. Micioni Di Bonaventura MV, et al. N/OFQ-NOP system in food intake. Handb Exp Pharmacol. 2019. https://doi. org/10.1007/164_2019_212.

86. Matsushita $\mathrm{H}$, et al. Chronic intracerebroventricular infusion of nociceptin/orphanin FQ produces body weight gain by affecting both feeding and energy metabolism in mice. Endocrinology. 2009. https://doi.org/10.1210/en. 2008-1515.

87. Webster CM, et al. Genome-wide RNAi screen for fat regulatory genes in $\mathrm{C}$. elegans identifies a proteostasisAMPK axis critical for starvation survival. Cell Rep. 2017. https://doi.org/10.1016/j.celrep.2017.06.068.

88. Azazmeh N, et al. Chronic expression of p16(INK4a) in the epidermis induces Wnt-mediated hyperplasia and promotes tumor initiation. Nat Commun. 2020. https://doi. org/10.1038/s41467-020-16475-3.

89. Yosef R, et al. Directed elimination of senescent cells by inhibition of BCL-W and BCL-XL. Nat Commun. 2016. https://doi.org/10.1038/ncomms11190.

90. Lim S, et al. Local delivery of senolytic drug inhibits intervertebral disc degeneration and restores intervertebral disc structure. Adv Healthc Mater. 2021. https://doi.org/ 10.1002/adhm.202101483.

Publisher's note Springer Nature remains neutral with regard to jurisdictional claims in published maps and institutional affiliations. 